\title{
Higgs mass and vacuum stability in the Standard Model at NNLO
}

\author{
Giuseppe Degrassi, ${ }^{a}$ Stefano Di Vita, ${ }^{a}$ Joan Elias-Miró, ${ }^{b}$ José R. Espinosa, ${ }^{b, c}$ \\ Gian F. Giudice, ${ }^{d}$ Gino Isidori $^{d, e}$ and Alessandro Strumia ${ }^{g, h}$ \\ ${ }^{a}$ Dipartimento di Fisica, Università di Roma Tre and INFN - Sezione di Roma Tre, \\ Roma, Italy \\ ${ }^{b}$ IFAE, Universitat Autónoma de Barcelona, \\ 08193 Bellaterra, Barcelona, Spain \\ ${ }^{c}$ ICREA, Instituciò Catalana de Recerca i Estudis Avançats, \\ Barcelona, Spain \\ ${ }^{d}$ CERN, Theory Division, \\ CH-1211 Geneva 23, Switzerland \\ e INFN, Laboratori Nazionali di Frascati, \\ Via E. Fermi 40, Frascati, Italy \\ ${ }^{g}$ Dipartimento di Fisica, Università di Pisa and INFN - Sezione di Pisa, \\ Pisa, Italy \\ ${ }^{h}$ National Institute of Chemical Physics and Biophysics, \\ Tallinn, Estonia \\ E-mail: degrassi@s.uniroma3.it, divita@s.uniroma3.it, jelias@ifae.es, \\ jose.ramon.espinosa.sedano@cern.ch, gian.giudice@cern.ch, \\ gino.isidori@lnf.infn.it, astrumia@mail.df .unipi.it
}

Abstract: We present the first complete next-to-next-to-leading order analysis of the Standard Model Higgs potential. We computed the two-loop QCD and Yukawa corrections to the relation between the Higgs quartic coupling $(\lambda)$ and the Higgs mass $\left(M_{h}\right)$, reducing the theoretical uncertainty in the determination of the critical value of $M_{h}$ for vacuum stability to $1 \mathrm{GeV}$. While $\lambda$ at the Planck scale is remarkably close to zero, absolute stability of the Higgs potential is excluded at $98 \%$ C.L. for $M_{h}<126 \mathrm{GeV}$. Possible consequences of the near vanishing of $\lambda$ at the Planck scale, including speculations about the role of the Higgs field during inflation, are discussed.

Keywords: Higgs Physics, Standard Model, Beyond Standard Model

ARXIV EPRINT: 1205.6497 


\section{Contents}

1 Introduction 1

2 The two-loop threshold correction to $\lambda(\mu) \quad 3$

2.1 Two-loop corrected Higgs mass from the effective potential 4

2.2 Two-loop contribution to $\Delta \lambda(\mu)$ in the gauge-less SM 7

3 Extrapolating the SM up to the Planck scale 11

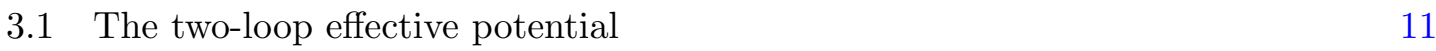

$\begin{array}{lll}3.2 & \text { Inputs at the electroweak scale and threshold corrections } & 13\end{array}$

$\begin{array}{lll}3.3 & \text { Phase diagram of the SM } & 15\end{array}$

$\begin{array}{llr}4 & \text { Implications } & \mathbf{1 7}\end{array}$

$\begin{array}{lll}4.1 & \text { Boundary conditions at the Planck scale } & 17\end{array}$

$\begin{array}{lll}4.2 & \text { Higgs inflation from non-minimal coupling to gravity } & 18\end{array}$

$\begin{array}{ll}4.3 \text { Higgs inflation from false vacuum } & 19\end{array}$

$\begin{array}{lll}4.4 & \text { Supersymmetry } & 21\end{array}$

5 Conclusions 23

A SM effective potential up to two-loops $\quad 26$

\section{Introduction}

The value of the Higgs mass $\left(M_{h}\right)$ favored by present ATLAS and CMS data [1, 2], $M_{h} \approx$ $125-126 \mathrm{GeV}$, is intriguing: it is quite close to the minimum $M_{h}$ value that ensures absolute vacuum stability within the Standard Model (SM) which, in turn, implies a vanishing Higgs quartic coupling $(\lambda)$ around the Planck scale. In order to assess if the measured Higgs mass is compatible with such a peculiar condition, a precise computation is needed.

The study of the stability of the SM vacuum has a long history [3-5] (see also [6-8] and references therein). The state-of-the-art analyses before the latest LHC data were done at the next-to-leading order (NLO) level [9-17]. This is based on two-loop renormalizationgroup (RG) equations, one-loop threshold corrections at the electroweak scale (possibly improved with two-loop terms in the case of pure QCD corrections), and one-loop improved effective potential (see [18] for a numerically updated analysis).

With this paper all the ingredients necessary for a complete next-to-next-to-leading order (NNLO) analysis in the strong, top Yukawa and Higgs quartic couplings become available. In particular, complete three-loop beta functions for all the SM gauge couplings have been presented in [19], while the leading three-loop terms in the RG evolution of $\lambda$, 

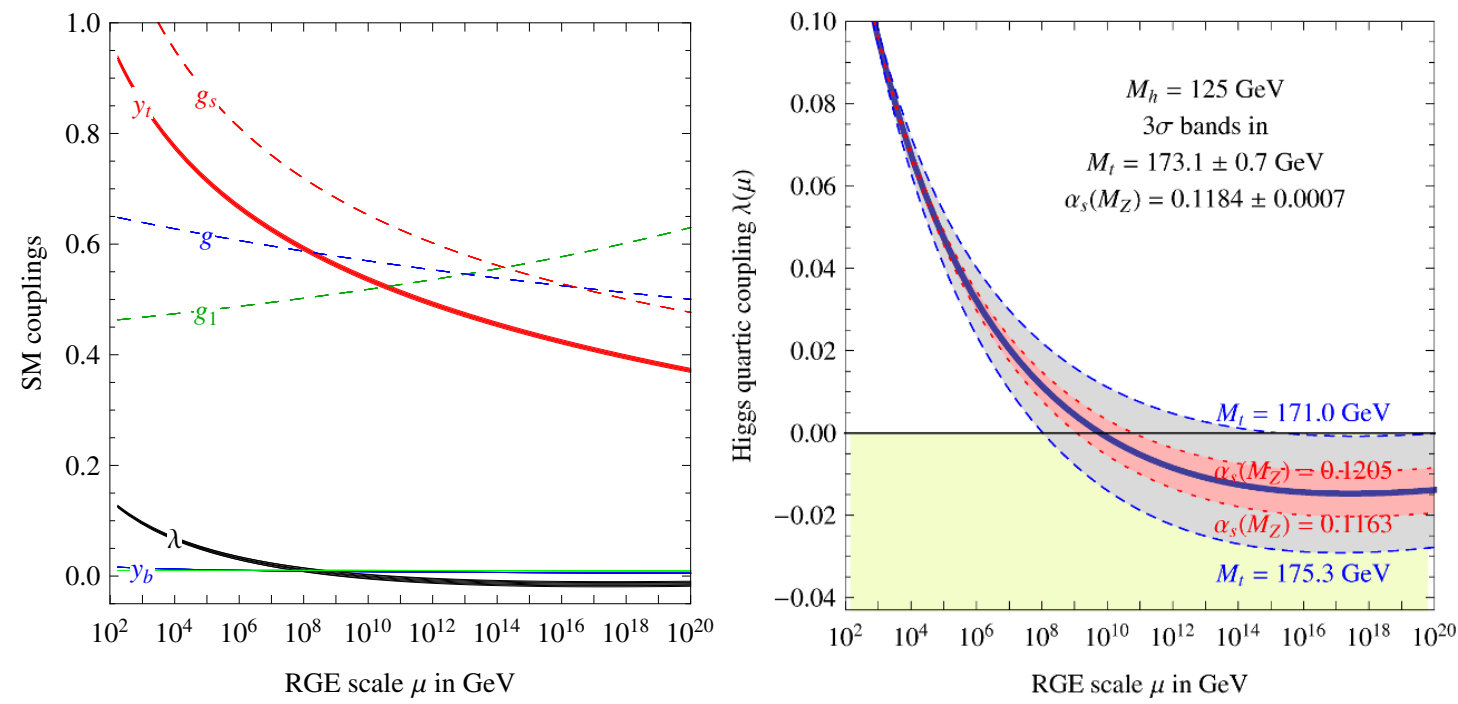

Figure 1. Left: SM RG evolution of the gauge couplings $g_{1}=\sqrt{5 / 3} g^{\prime}, g_{2}=g, g_{3}=g_{s}$, of the top and bottom Yukawa couplings $\left(y_{t}, y_{b}\right)$, and of the Higgs quartic coupling $\lambda$. All couplings are defined in the $\overline{\mathrm{MS}}$ scheme. The thickness indicates the $\pm 1 \sigma$ uncertainty. Right: RG evolution of $\lambda$ varying $M_{t}$ and $\alpha_{\mathrm{s}}$ by $\pm 3 \sigma$.

the top Yukawa coupling $\left(y_{t}\right)$ and the Higgs anomalous dimension have been computed in [20]. However, as pointed out in [18], the most important missing NNLO piece for the vacuum stability analysis are the two-loop threshold corrections to $\lambda$ at the weak scale due to QCD and top Yukawa interactions, because such couplings are sizable at low energy. The calculation of such terms is presented in this work.

The relation that connects $\lambda$ to the Higgs mass and to the the Fermi coupling $\left(G_{\mu}\right)$ can be written as

$$
\lambda(\mu)=\frac{G_{\mu} M_{h}^{2}}{\sqrt{2}}+\Delta \lambda(\mu),
$$

where $\Delta \lambda(\mu)$ denotes the sizable threshold corrections arising beyond the tree level. Given the rapid variation of $\lambda$ around the weak scale (see figure 1), these corrections play a significant role in determining the evolution of $\lambda$ up to high energies. Computing $\Delta \lambda(\mu)$ at the one loop level, using two-loop beta functions for all the SM couplings, and varying the low-energy matching scale between $M_{t} / 2$ and $2 M_{t}$, leads to a $\pm 2 \mathrm{GeV}$ error on $M_{h}$ [18]. The NNLO finite terms that we compute here allow us to reduce this error down to $\pm 0.7 \mathrm{GeV}$. While this work was in progress an independent calculation of the two-loop Yukawa-QCD contributions to $\Delta \lambda(\mu)$ has appeared [21]. Our result agrees with the one in ref. [21] for these contributions. However, our analysis includes also the two-loop terms coming from the Yukawa sector and can be considered the first complete NNLO evaluation of $\Delta \lambda(\mu)$. We stress that both these two-loop terms are needed to match the sizable two-loop scale dependence of $\lambda$ around the weak scale, caused by the $-32 y_{t}^{4} g_{s}^{2}+30 y_{t}^{6}$ terms in its beta function. As a result of this improved determination of $\Delta \lambda(\mu)$, we are able to obtain a significant reduction of the theoretical error on $M_{h}$ compared to previous works. 
Putting all the NNLO ingredients together, we estimate an overall theory error on $M_{h}$ of $\pm 1.0 \mathrm{GeV}$ (see section 3). Our final results for the condition of absolute stability up to the Planck scale is

$$
M_{h}[\mathrm{GeV}]>129.4+1.4\left(\frac{M_{t}[\mathrm{GeV}]-173.1}{0.7}\right)-0.5\left(\frac{\alpha_{s}\left(M_{Z}\right)-0.1184}{0.0007}\right) \pm 1.0_{\mathrm{th}} .
$$

Combining in quadrature the theoretical uncertainty with the experimental errors on $M_{t}$ and $\alpha_{s}$ we get

$$
M_{h}>129.4 \pm 1.8 \mathrm{GeV}
$$

From this result we conclude that vacuum stability of the SM up to the Planck scale is excluded at $2 \sigma$ (98\% C.L. one sided) for $M_{h}<126 \mathrm{GeV}$.

Although the central values of Higgs and top masses do not favor a scenario with a vanishing Higgs self coupling at the Planck scale $\left(M_{\mathrm{Pl}}\right)$ - a possibility originally proposed in ref. [22, 23] and discussed more recently in ref. [12, 13, 15, 24, 25] — the smallness of $\lambda$ around $M_{\mathrm{Pl}}$ is quite remarkable (see figure 1). Motivated by this observation, we have explored in more detail the robustness of the predictions for $M_{h}$ and $M_{t}$ assuming special boundary conditions on $\lambda$ and its beta function around $M_{\mathrm{Pl}}$, as advocated in [24]. We have also critically examined scenarios where the Higgs field plays a role during inflation. This could happen because of a non-minimal coupling to gravity that flattens the SM potential close to $M_{\mathrm{Pl}}$ [15], provided $\lambda\left(M_{\mathrm{Pl}}\right)$ is positive. Alternatively, the Higgs field could have caused inflation while it was trapped in a second unstable minimum of the potential that appears near $M_{\mathrm{Pl}}$ if $\lambda\left(M_{\mathrm{Pl}}\right)$ is positive and very close to zero [13], provided some non-SM mechanism is introduced to exit inflation [26, 27]. Beside some technical problems, these frameworks could become viable only if $M_{h}$ satisfies the stability condition in eq. (1.2). We therefore conclude that both these possibilities are not favored by present data, unless $M_{t}$ is below about $172 \mathrm{GeV}$ or new-physics threshold corrections at the high scale modify the shape of the SM potential.

The paper is organized as follows: in section 2 we present the calculation of the threshold correction $\Delta \lambda(\mu)$. The numerical results for the condition of vacuum stability and, more generally, for the structure of the SM Higgs potential up to very high field values are discussed in section 3. The implications of these results for Planck scale physics are discussed in section 4. The results are summarized in the conclusions. We include also an appendix in which a ready-to-use expression for the two-loop effective potential is presented.

\section{The two-loop threshold correction to $\lambda(\mu)$}

In this section we present our main new result, namely the calculation of the two-loop contribution to $\Delta \lambda(\mu)$. We first obtain the $y_{t}^{6}$ and $y_{t}^{4} g_{s}^{2}$ terms from the calculation of the Higgs mass via the effective potential. Then, we present the full result for the two-loop QCD and Yukawa contribution to $\Delta \lambda(\mu)$ in the SM with the electroweak gauge couplings switched off (the so-called gauge-less limit). 


\subsection{Two-loop corrected Higgs mass from the effective potential}

We write the SM potential for the Higgs doublet $H$ in the usual way:

$$
V=-m^{2}|H|^{2}+\lambda|H|^{4} \quad H=\left(\begin{array}{c}
G^{+} \\
\left(v+h+i G^{0}\right) / \sqrt{2}
\end{array}\right),
$$

so that, up to negligible width effects, the pole Higgs mass $M_{h}$ is the solution of the pole equation

$$
M_{h}^{2}=-m^{2}+3 \lambda v^{2}+\Pi_{h h}\left(M_{h}^{2}\right),
$$

where $m^{2}, \lambda$ and $v$ are $\overline{\mathrm{MS}}$ renormalized quantities and $\Pi_{h h}\left(p^{2}\right)$ is the Higgs self-energy (two-point) function, with external four-momentum $p$. We rewrite this equation as

$$
\begin{aligned}
M_{h}^{2} & =\left[-m^{2}+3 \lambda v^{2}+\Pi_{h h}(0)\right]+\left[\Pi_{h h}\left(M_{h}^{2}\right)-\Pi_{h h}(0)\right] \\
& =\left[M_{h}^{2}\right]_{V}+\Delta \Pi_{h h}\left(M_{h}^{2}\right) .
\end{aligned}
$$

This step is convenient because the last term (which is computationally challenging) only gives corrections suppressed by the small Higgs coupling, in view of the smallness of $M_{h}^{2}=$ $2 \lambda v^{2}$ at tree level. The first dominant term can be expressed in term of derivatives of the effective potential, $V_{\text {eff }}$. Writing the effective potential as a sum of the tree-level part $V_{0}$ plus radiative corrections $\Delta V$

$$
V_{\mathrm{eff}}=-\frac{m^{2}}{2} h^{2}+\frac{\lambda}{4} h^{4}+\Delta V
$$

one finds

$$
\left[M_{h}^{2}\right]_{V}=\left.\frac{\partial^{2} V_{\mathrm{eff}}}{(\partial h)^{2}}\right|_{h=v},
$$

where $v$ is the $h$ vev at the minimum of the effective potential, determined by the minimization condition

$$
\left.\frac{\partial V_{\mathrm{eff}}}{\partial h}\right|_{h=v}=\left[-m^{2} h+\lambda h^{3}+\frac{\partial \Delta V}{\partial h}\right]_{h=v} .
$$

As usual, it is convenient to consider $m^{2}$ as a free parameter fixed in terms of $v$ by the above equation, arriving at

$$
\left[M_{h}^{2}\right]_{V}=\left[2 \lambda v^{2}-\frac{1}{h} \frac{\partial \Delta V}{\partial h}+\frac{\partial^{2} \Delta V}{(\partial h)^{2}}\right]_{h=v} .
$$

Defining the operator $\mathcal{D}_{m}^{2}$ as ${ }^{1}$

$$
\mathcal{D}_{m}^{2}=\left[-\frac{1}{h} \frac{\partial}{\partial h}+\frac{\partial^{2}}{(\partial h)^{2}}\right]_{h=v},
$$

and noting that $2 \lambda v^{2}=\mathcal{D}_{m}^{2} V_{0}$, we can simply write $\left[M_{h}^{2}\right]_{V}=\mathcal{D}_{m}^{2} V_{\text {eff }}$, obtaining the following form for the Higgs mass:

$$
M_{h}^{2}=\mathcal{D}_{m}^{2} V_{\text {eff }}+\Delta \Pi_{h h}\left(M_{h}^{2}\right) .
$$

\footnotetext{
${ }^{1}$ Notice that the term in $\mathcal{D}_{m}^{2}$ linear in field-derivatives automatically takes into account the cancellation of $h$-tadpoles (or alternatively, the minimization condition to get the right $v$ ).
} 
It gives the Higgs mass squared as the sum of two terms. The first is the Higgs mass obtained from the potential; this is not the complete pole Higgs mass and must be corrected for nonzero external momentum effects, which are taken care of by the last term, $\Delta \Pi_{h h}\left(M_{h}^{2}\right)$. It is a straightforward exercise to verify that this expression for pole mass is independent of the renormalization scale $\mu$. In particular, one can easily prove that

$$
\begin{aligned}
\frac{d}{d \ln \mu}\left[M_{h}^{2}\right]_{V} & =-2 \gamma\left[M_{h}^{2}\right]_{V}, \\
\frac{d}{d \ln \mu} \Delta \Pi_{h h}\left(M_{h}^{2}\right) & =2 \gamma\left[M_{h}^{2}-\Delta \Pi_{h h}\left(M_{h}^{2}\right)\right],
\end{aligned}
$$

where $\gamma$ is the Higgs anomalous dimension, describing its wave-function renormalization, $\gamma \equiv d \ln h / d \ln \mu$.

Using eq. (2.9) and the one-loop result for $V_{\text {eff }}$ in eq. (A.2) of the appendix one obtains the one-loop Higgs mass correction. The explicit one-loop result for the pole mass is

$$
M_{h}^{2}=2 \lambda v^{2}+\delta_{1} M_{h}^{2}
$$

with

$$
\begin{aligned}
\delta_{1} M_{h}^{2}=\frac{1}{(4 \pi)^{2}}\left\{3 y_{t}^{2}(\right. & \left.4 m_{t}^{2}-M_{h}^{2}\right) B_{0}\left(m_{t}, m_{t}, M_{h}\right)+6 \lambda^{2} v^{2}\left(3 \ell_{h}-6+\pi \sqrt{3}\right) \\
& -\frac{v^{2}}{4}\left(3 g^{4}-8 \lambda g^{2}+16 \lambda^{2}\right) B_{0}\left(m_{W}, m_{W}, M_{h}\right) \\
& -\frac{v^{2}}{8}\left(3 G^{4}-8 \lambda G^{2}+16 \lambda^{2}\right) B_{0}\left(m_{Z}, m_{Z}, M_{h}\right) \\
+ & \left.2 m_{W}^{2}\left[g^{2}-2 \lambda\left(\ell_{W}-1\right)\right]+m_{Z}^{2}\left[G^{2}-2 \lambda\left(\ell_{Z}-1\right)\right]\right\},
\end{aligned}
$$

where $G^{2}=g^{2}+g^{\prime 2}$. All parameters on the right-hand side (including $v$ ) are $\overline{\mathrm{MS}}$ running parameters (with the exception of $M_{h}^{2}$, which appears through the external momentum dependence of the Higgs self-energy). As eq. (A.2) was obtained in the Landau gauge, $v$ in eq. (2.13) represents the gauge and scale-dependent vacuum expectation value of the Higgs field as computed in the Landau gauge. Similarly the $\Delta \Pi_{h h}\left(M_{h}^{2}\right)$ contribution in that equation is computed in the Landau gauge. In eq. (2.13)

$$
B_{0}\left(m_{a}, m_{b}, m_{c}\right) \equiv-\int_{0}^{1} \ln \frac{(1-x) m_{a}^{2}+x m_{b}^{2}-x(1-x) m_{c}^{2}-i \epsilon}{\mu^{2}} d x
$$

and $\ell_{x} \equiv \ln \left(m_{x}^{2} / \mu^{2}\right)$, with $m_{x}$ the running mass for particle $x\left(m_{t} \equiv y_{t} v / \sqrt{2}\right)$. One can explicitly check, using the RGEs for these parameters, that this expression for $M_{h}^{2}$ is indeed scale-independent at one-loop order.

Neglecting gauge couplings and setting $M_{h}^{2}=2 \lambda v^{2}$ in the one-loop terms, one obtains the approximate expression

$$
\delta_{1} M_{h}^{2} \simeq \frac{2 y_{t}^{2} v^{2}}{(4 \pi)^{2}}\left[\lambda\left(2+3 \ell_{t}\right)-3 y_{t}^{2} \ell_{t}\right]
$$



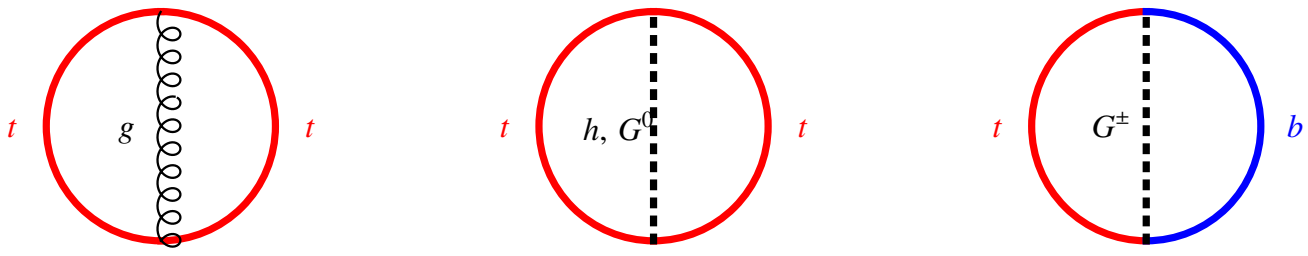

Figure 2. Two-loop vacuum diagrams that give the dominant contribution (depending only on $g_{s}$ and $\left.y_{t}\right)$ to the SM two-loop effective potential.

To compute eq. (2.9) at the two-loop level one can use the two-loop effective potential $[28,29]$ to calculate $\left[M_{h}^{2}\right]_{V}$ and the general results for two-loop scalar self-energies in [30] (supplemented by the results on two-loop momentum integrals of [31]) to calculate $\Delta \Pi_{h h}\left(M_{h}^{2}\right)$. If we only keep the leading two-loop corrections to $M_{h}^{2}$ proportional to $y_{t}^{6}$, $y_{t}^{4} g_{s}^{2}$, dropping all subleading terms that depend on the electroweak gauge couplings or $\lambda$, our task is simplified dramatically. First, in the two-loop effective potential we only have to consider the diagrams depicted in figure 2. Their contribution can be extracted from the expressions for $V_{Y}$ and $V_{F V}$ in the appendix. Second, in the two-loop term $\Delta \Pi_{h h}^{(2)}\left(M_{h}^{2}\right)$ we can substitute the tree-level value $M_{h}^{2}=2 \lambda v^{2}$, so that

$$
\Delta \Pi_{h h}^{(2)}\left(M_{h}^{2}\right) \simeq \Pi_{h h}^{(2)}\left(2 \lambda v^{2}\right)-\Pi_{h h}^{(2)}(0) .
$$

It is then clear that the two-loop contributions coming from that term are proportional to $\lambda$ and are therefore subdominant. In this section we neglect $\Delta \Pi_{h h}^{(2)}\left(M_{h}^{2}\right)$ completely.

To find the expression for the Higgs mass at two-loop precision, we must also take into account that $M_{h}^{2}$ has to be evaluated with one-loop precision in the argument of the one-loop term $\Delta \Pi_{h h}^{(1)}\left(M_{h}^{2}\right)$. Putting together all these pieces, keeping only the $y_{t}^{6}$ and $y_{t}^{4} g_{s}^{2}$ terms, we arrive at the following two-loop correction to eq. (2.12):

$$
\delta_{2} M_{h}^{2}=\frac{y_{t}^{2} v^{2}}{(4 \pi)^{4}}\left[16 g_{s}^{2} y_{t}^{2}\left(3 \ell_{t}^{2}+\ell_{t}\right)-3 y_{t}^{4}\left(9 \ell_{t}^{2}-3 \ell_{t}+2+\frac{\pi^{2}}{3}\right)\right] .
$$

The expression for $M_{h}$ as a function of $\lambda$ can be inverted to obtain $\lambda(\mu)$ as a function of the pole Higgs mass $M_{h}$. To express $\lambda(\mu)$ in terms of physical quantities (i.e. $G_{\mu}$ and the pole masses $M_{Z}, M_{W}$, and $M_{t}$ ) the relations between physical and $\overline{\mathrm{MS}}$ parameters are needed. At the level of accuracy we are working only the relation between the $y_{t}(\mu)$ and $M_{t}$ and the one between $v(\mu)$ and $G_{\mu}$ are required. They are given by:

$$
\begin{aligned}
y_{t}^{2}(\mu)=2 \sqrt{2} G_{\mu} M_{t}^{2}\left[1+\frac{8}{3} \frac{1}{(4 \pi)^{2}} g_{s}^{2}\left(3 L_{T}-4\right)+\frac{1}{(4 \pi)^{2}} \sqrt{2} G_{\mu} M_{t}^{2}\left(-9 L_{T}+11\right)\right], \\
v^{2}(\mu)=\frac{1}{\sqrt{2} G_{\mu}}+\frac{1}{(4 \pi)^{2}}\left[3 M_{t}^{2}\left(2 L_{T}-1\right)+M_{W}^{2}\left(5-6 L_{W}\right)+\frac{1}{2} M_{Z}^{2}\left(5-6 L_{Z}\right)\right. \\
\left.+\frac{3 M_{Z}^{2} M_{W}^{2}}{4\left(M_{Z}^{2}-M_{W}^{2}\right)}\left(L_{Z}-L_{W}\right)-\frac{1}{2} M_{h}^{2}-\frac{3 M_{W}^{2} M_{h}^{2}}{M_{W}^{2}-M_{h}^{2}}\left(L_{W}-L_{H}\right)\right],
\end{aligned}
$$

where $L_{X}=\ln \left(M_{x}^{2} / \mu^{2}\right)$, with masses in capital letters denoting pole masses. 
We find:

$$
\lambda(\mu)=\frac{G_{\mu} M_{h}^{2}}{\sqrt{2}}+\lambda^{(1)}(\mu)+\lambda^{(2)}(\mu)
$$

with

$$
\lambda^{(2)}(\mu)=\lambda_{\mathrm{QCD}, \text { lead. }}^{(2)}(\mu)+\lambda_{\text {Yuk,lead. }}^{(2)}(\mu)+\ldots,
$$

where the ellipsis stands for the subleading terms neglected in this section. The known one loop term is

$$
\begin{aligned}
\lambda^{(1)}(\mu)=\frac{1}{2} G_{\mu}^{2} & \frac{1}{(4 \pi)^{2}}\left\{\frac{6\left(L_{H}-L_{W}\right) M_{h}^{6}}{M_{h}^{2}-M_{W}^{2}}-8\left(2 M_{W}^{4}+M_{Z}^{4}\right)-2\left(-3+6 L_{T}\right) M_{h}^{2} M_{t}^{2}\right. \\
& +M_{h}^{4}\left(19-15 L_{H}+6 L_{W}-3 \sqrt{3} \pi\right)+12\left(M_{h}^{2}-4 M_{t}^{2}\right) M_{t}^{2} B_{0}\left(M_{t}, M_{t}, M_{h}\right) \\
& +2\left(M_{h}^{4}-4 M_{h}^{2} M_{W}^{2}+12 M_{W}^{4}\right) B_{0}\left(M_{W}, M_{W}, M_{h}\right) \\
& +\left(M_{h}^{4}-4 M_{h}^{2} M_{Z}^{2}+12 M_{Z}^{4}\right) B_{0}\left(M_{Z}, M_{Z}, M_{h}\right) \\
& \left.+M_{h}^{2}\left[2\left(8 L_{W}-7\right) M_{W}^{2}+\left(8 L_{Z}-7\right) M_{Z}^{2}-\frac{6 M_{Z}^{2} M_{W}^{2}}{M_{Z}^{2}-M_{W}^{2}}\left(L_{Z}-L_{W}\right)\right]\right\},
\end{aligned}
$$

and the leading two loop QCD and Yukawa terms are

$$
\begin{aligned}
\lambda_{\mathrm{QCD}, \text { lead. }}^{(2)}(\mu) & =\frac{G_{\mu}^{2} M_{t}^{4}}{(4 \pi)^{4}} 64 g_{s}^{2}(\mu)\left(-4-6 L_{T}+3 L_{T}^{2}\right), \\
\lambda_{\text {Yuk,lead. }}^{(2)}(\mu) & =\frac{8 \sqrt{2} G_{\mu}^{3} M_{t}^{6}}{(4 \pi)^{4}}\left(30+\pi^{2}+36 L_{T}-45 L_{T}^{2}\right) .
\end{aligned}
$$

The above expression for $\lambda(\mu)$ has the correct dependence on the renormalization scale $\mu$, so that both sides of (2.20) evolve with $\mu$ in the same way to the order we work.

\subsection{Two-loop contribution to $\Delta \lambda(\mu)$ in the gauge-less SM}

In this section we go beyond the leading $y_{t}^{6}$ and $y_{t}^{4} g_{s}^{2}$ contributions to $\Delta \lambda(\mu)$ discussed in the previous section and derive the QCD and Yukawa corrections in the SM with the electroweak gauge couplings switched off. We first discuss the two-loop renormalization of the Higgs sector of the SM and then derive the 2-loop relation between $\lambda(\mu)$ and the physical parameters $G_{\mu}, M_{t}$, and $M_{h}$. In our derivation we follow closely ref. [32].

We start from the unrenormalized Higgs potential, eq. (2.1) written in terms of bare quantities, and set $m^{2} \rightarrow m_{r}^{2}-\delta m^{2}, \lambda \rightarrow \lambda_{r}-\delta \lambda, v \rightarrow v_{r}-\delta v$. Then, assuming $\delta m^{2}, \delta \lambda, \delta v$ of $\mathcal{O}(\alpha)$, we obtain

$$
V=V_{r}-\delta V
$$

where, putting $m_{r}^{2}=\lambda_{r} v_{r}^{2}$,

$$
\begin{aligned}
V_{r}= & \lambda_{r}\left[G^{+} G^{-}\left(G^{+} G^{-}+h^{2}+G_{0}\right)^{2}+\frac{1}{4}\left(h^{2}+G_{0}^{2}\right)^{2}\right] \\
& +\lambda_{r} v_{r} h\left[h^{2}+G_{0}^{2}+2 G^{+} G^{-}\right]+\frac{1}{2} M_{h}^{2} h^{2},
\end{aligned}
$$


with $M_{h}=2 \lambda_{r} v_{r}^{2}$, while, up to two-loop terms,

$$
\begin{aligned}
\delta V= & \delta \lambda\left[G^{+} G^{-}\left(G^{+} G^{-}+h^{2}+G_{0}\right)^{2}+\frac{1}{4}\left(h^{2}+G_{0}^{2}\right)^{2}\right] \\
& +\left[\lambda_{r}\left(\frac{\delta v^{2}}{2 v_{r}}+\frac{\left(\delta v^{2}\right)^{2}}{8 v_{r}^{3}}\right)+v_{r} \delta \lambda\left(1-\frac{\delta v^{2}}{2 v_{r}^{2}}\right)\right] h\left[h^{2}+G_{0}^{2}+2 G^{+} G^{-}\right] \\
& +\delta \tau\left(\frac{1}{2} G_{0}^{2}+G^{+} G^{-}\right)+\frac{1}{2} \delta M_{h}^{2} h^{2}+v_{r} \delta \tau\left(1-\frac{\delta v^{2}}{2 v_{r}^{2}}\right) h .
\end{aligned}
$$

In eq. $(2.27)$

$$
\begin{gathered}
\delta M_{h}^{2}=3\left[\lambda_{r} \delta v^{2}+v_{r}^{2} \delta \lambda\left(1-\frac{\delta v^{2}}{v_{r}^{2}}\right)\right]-\delta m^{2}, \\
\delta \tau=\lambda_{r} \delta v^{2}+v_{r}^{2} \delta \lambda\left(1-\frac{\delta v^{2}}{v_{r}^{2}}\right)-\delta m^{2}
\end{gathered}
$$

and $\delta v^{2}$ is related to $\delta v$ through $\sqrt{v_{r}^{2}-\delta v^{2}}=v_{r}-\delta v$.

Following ref. [32] we require the cancellation of the tadpole contribution by setting

$$
\delta \tau\left(1-\frac{\delta v^{2}}{2 v_{r}^{2}}\right)=-\frac{T}{v_{r}}
$$

where $i T$ is the sum of the tadpole diagrams with the external leg extracted. We identify $M_{h}^{2}$ in $V_{r}$ with the on-shell Higgs mass leading to the condition

$$
\delta M_{h}^{2}=\operatorname{Re} \Pi_{h h}\left(M_{h}^{2}\right),
$$

where the $\Pi_{h h}\left(M_{h}^{2}\right)$ in the above equation includes only the contribution of the self-energy diagrams because eq. (2.30) is enforced. A third condition can be obtained by looking directly at the muon-decay process. At the two-loop level we can write

$$
\frac{G_{\mu}}{\sqrt{2}}=\frac{1}{2 v_{0}^{2}}\left\{1-\frac{A_{W W}}{M_{W_{0}}^{2}}+V_{W}+M_{W_{0}}^{2} B_{W}+\left(\frac{A_{W W}}{M_{W}^{2}}\right)^{2}-\frac{A_{W W} V_{W}}{M_{W}^{2}}\right\},
$$

where $v_{0}$ is the unrenormalized vacuum, $A_{W W} \equiv A_{W W}(0)$ is the $W$ self-energy evaluated at zero external momenta, $V_{W}$ and $B_{W}$ are the relevant vertex and box contributions in the $\mu$-decay process and $M_{W_{0}}$ is the unrenormalized $W$ mass. Performing the shifts $v_{0}^{2} \rightarrow v_{r}^{2}-\delta v^{2}, M_{W_{0}} \rightarrow M_{W}^{2}-\delta M_{W}^{2}$, where $\delta M_{W}^{2}=\operatorname{Re} A_{W W}\left(M_{W}^{2}\right)$, and working at the two-loop level we arrive at

$$
\begin{aligned}
v_{r}^{2}=\frac{1}{\sqrt{2} G_{\mu}}-\frac{1}{\sqrt{2} G_{\mu}}\left\{\frac{A_{W W}}{M_{W}^{2}}-E+\frac{A_{W W} \delta M_{W}^{2}}{M_{W}^{4}}\right. \\
\left.\quad-\left(\frac{A_{W W}}{M_{W}^{2}}\right)^{2}+\frac{A_{W W} V_{W}}{M_{W}^{2}}+\delta M_{W}^{2} B_{W}\right\}+\delta v^{2},
\end{aligned}
$$

where $E \equiv V_{W}+M_{W}^{2} B_{W}$. We identify the renormalized vacuum by $v_{r}^{2}=1 /\left(\sqrt{2} G_{\mu}\right)$, then $\delta v^{2}$ is defined to cancel the contribution of the curly bracket in eq. (2.33). 
Our choice of renormalization conditions implies that the renormalized quartic Higgs coupling is set equal to

$$
\lambda_{r}=\frac{G_{\mu}}{\sqrt{2}} M_{h}^{2},
$$

while eqs. (2.28)-(2.33) can be used to obtain the correction $\delta \lambda$. Writing

$$
\delta \lambda=\delta \lambda^{(1)}+\delta \lambda^{(2)},
$$

where the superscript indicates the loop order, we have

$$
\begin{aligned}
\delta \lambda^{(1)}=-\frac{G_{\mu}}{\sqrt{2}} M_{h}^{2} & \left\{\frac{A_{W W}^{(1)}}{M_{W}^{2}}-E^{(1)}-\frac{1}{M_{h}^{2}}\left[\operatorname{Re} \Pi_{h h}^{(1)}\left(M_{h}^{2}\right)+\frac{T^{(1)}}{v_{r}}\right]\right\} \\
\delta \lambda^{(2)}=-\frac{G_{\mu}}{\sqrt{2}} M_{h}^{2} & \left\{\frac{A_{W W}^{(2)}}{M_{W}^{2}}-E^{(2)}-\frac{1}{M_{h}^{2}}\left[\operatorname{Re} \Pi_{h h}^{(2)}\left(M_{h}^{2}\right)+\frac{T^{(2)}}{v_{r}}\right]\right. \\
& +\left(\frac{A_{W W}^{(1)}}{M_{W}^{2}}-E^{(1)}\right)\left(\frac{A_{W W}^{(1)}}{M_{W}^{2}}-E^{(1)}-\frac{1}{M_{h}^{2}}\left[\operatorname{Re} \Pi_{h h}^{(1)}\left(M_{h}^{2}\right)+\frac{3}{2} \frac{T^{(1)}}{v_{r}}\right]\right) \\
& \left.+\frac{A_{W W}^{(1)} \delta^{(1)} M_{W}^{2}}{M_{W}^{4}}-\left(\frac{A_{W W}^{(1)}}{M_{W}^{2}}\right)^{2}+\frac{A_{W W}^{(1)} V_{W}^{(1)}}{M_{W}^{2}}+\delta^{(1)} M_{W}^{2} B_{W}^{(1)}\right\} .
\end{aligned}
$$

The connection between $\lambda_{r}$, as defined in eq. (2.34), and $\lambda(\mu)$ can be easily derived using

$$
\lambda_{r}-\delta \lambda=\lambda(\mu)-\delta \hat{\lambda}
$$

or

$$
\lambda(\mu)=\frac{G_{\mu}}{\sqrt{2}} M_{h}^{2}-\delta \lambda+\delta \hat{\lambda} .
$$

In eqs. (2.38)-(2.39) $\delta \hat{\lambda}$ is the counterterm associated to $\lambda(\mu)$, i.e. the counterterm that subtracts only the terms proportional to powers of $1 / \epsilon$ and $\gamma-\ln (4 \pi)$ in dimensional regularization, with $d=4-2 \epsilon$ being the dimension of space-time. Concerning the structure of the $1 / \epsilon$ poles in $\delta \lambda$ and $\delta \hat{\lambda}$, one notices that it should be identical once the poles in $\delta \lambda$ are expressed in terms of $\overline{\mathrm{MS}}$ quantities. Then, after this operation is performed, a finite $\lambda(\mu)$ is obtained.

Specializing the above discussion to the two-loop case we have

$$
\lambda(\mu)=\frac{G_{\mu}}{\sqrt{2}} M_{h}^{2}-\left.\delta \lambda^{(1)}\right|_{\text {fin }}-\left.\delta \lambda^{(2)}\right|_{\text {fin }}+\Delta
$$

from which we identify the one- and two-loop contributions entering eq. (2.20),

$$
\lambda^{(1)}(\mu)=-\left.\delta \lambda^{(1)}\right|_{\text {fin }},
$$

which reproduces the one-loop result of ref. [32] and

$$
\lambda^{(2)}(\mu)=-\left.\delta \lambda^{(2)}\right|_{\text {fin }}+\Delta .
$$


In eqs. (2.40)-(2.42) the subscript 'fin' denotes the finite part of the quantity involved and $\Delta$ is the two-loop finite contribution that is obtained when the OS parameters entering the $1 / \epsilon$ pole in $\delta \lambda^{(1)}$ are expressed in terms of $\overline{\mathrm{MS}}$ quantities, the finite contribution coming from the $O(\epsilon)$ part of the shifts.

Differentiating eq. (2.40) with respect to $\mu$, the known two-loop beta function for the Higgs quartic coupling is recovered. It should be recalled that the right-hand side of eq. (2.40) is expressed in terms of physical quantities, then the dependence on $\mu$ in that equation is explicit. To obtain the correct two-loop beta function, one has first to differentiate with respect to $\mu$ and then to express the one-loop part in terms of $\overline{\mathrm{MS}}$ quantities.

The computation of $\lambda^{(2)}(\mu)$ in the full SM is quite cumbersome, see eq. (2.37). However, the calculation can be greatly simplified if one considers the gauge-less limit of the SM in which the electroweak interactions are neglected, i.e. the gauge couplings $g$ and $g^{\prime}$ are set equal to zero. In this limit, eq. (2.37) simplifies to

$$
\begin{aligned}
\left(\delta \lambda^{(2)}-\Delta\right)_{\text {g.l. }}=-\frac{G_{\mu}}{\sqrt{2}} & M_{h}^{2}\left\{\frac{A_{W W}^{(2)}}{M_{W}^{2}}-\frac{1}{M_{h}^{2}}\left[\operatorname{Re} \Pi_{h h}^{(2)}\left(M_{h}^{2}\right)+\frac{T^{(2)}}{v_{r}}\right]\right. \\
+ & \left.\frac{A_{W W}^{(1)}}{M_{W}^{2}}\left(\frac{A_{W W}^{(1)}}{M_{W}^{2}}-\frac{1}{M_{h}^{2}}\left[\operatorname{Re} \Pi_{h h}^{(1)}\left(M_{h}^{2}\right)+\frac{3}{2} \frac{T^{(1)}}{v_{r}}\right]\right)\right\}_{\text {g.l. }}-\Delta_{\text {g.l. }},
\end{aligned}
$$

where the subscript $g . l$. means that we have considered in the various self-energies only diagrams involving the top and bottom quarks, the Higgs and the Goldstone bosons, the latter with vanishing mass, and the limit $g, g^{\prime} \rightarrow 0$ is taken.

Using eq. (2.43) we compute the QCD and the Yukawa contribution to $\lambda^{(2)}(\mu)$. The top Yukawa-QCD contribution, $\lambda_{\mathrm{QCD}}^{(2)}(\mu)$, is obtained evaluating the relevant diagrams via a Taylor series in $x_{h t} \equiv M_{h}^{2} / M_{t}^{2}$ up to fourth order

$$
\begin{aligned}
\lambda_{\mathrm{QCD}}^{(2)}(\mu)= & \frac{G_{\mu}^{2} M_{t}^{4}}{(4 \pi)^{4}} N_{c} C_{F} g_{s}^{2}(\mu)\left[16\left(-4-6 L_{T}+3 L_{T}^{2}\right)\right. \\
& \left.+x_{h t}\left(35-\frac{2 \pi^{2}}{3}+12 L_{T}-12 L_{T}^{2}\right)+x_{h t}^{2} \frac{61}{135}+x_{h t}^{3} \frac{1223}{6300}+x_{h t}^{4} \frac{43123}{1323000}\right]
\end{aligned}
$$

where $N_{c}$ and $C_{F}$ are color factors $\left(N_{c}=3, C_{F}=4 / 3\right)$. Equation (2.44) shows that the series converges very fast. Our result is in agreement with ref. [21], the numerical difference between eq. (2.44) and the expression of ref. [21] for $M_{h} \sim 125 \mathrm{GeV}$ being negligible.

The Yukawa contribution, $\lambda_{\text {Yuk }}^{(2)}(\mu)$, is (neglecting the small bottom Yukawa)

$$
\begin{aligned}
& \lambda_{\text {Yuk }}^{(2)}(\mu)=\frac{\sqrt{2} G_{\mu}^{3} M_{t}^{6}}{(4 \pi)^{4}}\left\{N_{c}^{2}[\right. 16 B_{0}\left(M_{t}, M_{t}, M_{h}\right)\left(-1+2 L_{T}\right) \\
&\left.+x_{h t}\left(\left(1+4 B_{0}\left(M_{t}, M_{t}, M_{h}\right)-2 L_{T}\right)\left(1-2 L_{T}\right)\right)\right] \\
&+N_{c}\left[16+\frac{8}{3} \pi^{2}+\right. 32 B_{0}\left(M_{t}, M_{h}, M_{t}\right)\left(1+2 L_{T}\right)-48 L_{T}+40 L_{T}^{2} \\
&-x_{h t}\left(\frac{929}{6}+\frac{16}{3} \pi^{2}+48 B_{0}\left(M_{h}, M_{h}, M_{h}\right)-16 L_{H}\left(1-L_{T}\right)\right. \\
&\left.+B_{0}\left(M_{t}, M_{h}, M_{t}\right)\left(\frac{76}{3}+32 L_{T}\right)+\frac{190}{3} L_{T}+58 L_{T}^{2}\right)
\end{aligned}
$$




$$
\begin{gathered}
+x_{h t}^{2}\left(\frac{17629}{270}+\frac{8}{3} \pi^{2}-\frac{2}{3} L_{H}+B_{0}\left(M_{h}, M_{h}, M_{h}\right)\left(27-18 L_{T}\right)+40 L_{T}\right. \\
\left.+10 L_{T} L_{H}+12 L_{T}^{2}+B_{0}\left(M_{t}, M_{h}, M_{t}\right)\left(\frac{13}{3}+4 L_{T}\right)\right) \\
+x_{h t}^{3}\left(\frac{1181}{900}-\frac{\pi^{2}}{2}+\frac{61}{30} B_{0}\left(M_{h}, M_{h}, M_{h}\right)+\frac{59}{90} L_{H}\right. \\
\left.\left.-\frac{2}{35} B_{0}\left(M_{t}, M_{h}, M_{t}\right)-\frac{68}{63} L_{T}\right)\right] \\
+x_{h t}^{3}\left[\frac{131}{6} \pi^{2}+\left(\frac{729}{2}-\frac{135}{4} \sqrt{3} \pi\right) S_{2}-111 L_{H}+36 L_{H}^{2}\right. \\
\left.\left.+\pi\left(\frac{-225 \sqrt{3}}{4}+18 \sqrt{3} L_{H}\right)+\frac{75+72 \zeta_{3}}{4}\right]\right\}
\end{gathered}
$$

where $B_{0}\left(M_{h}, M_{h}, M_{h}\right)=2-L_{H}-\pi / \sqrt{3}$ and $S_{2}=4 /(9 \sqrt{3}) \mathrm{Cl}_{2}(\pi / 3)=0.260434138 \ldots$ In eq. (2.45) the terms proportional to $N_{c}^{2}$ and $N_{c}^{0}$ were computed exactly while the ones proportional to $N_{c}$ were computed via an asymptotic expansion in the large top mass up to $x_{h t}^{3}$ terms exploiting the asymptotic-expansion techniques developed in ref. [33]. The part independent of $N_{c}$ in eq. (2.45) was computed using the results for the two-loop on-shell master integrals of ref. [34].

We end this section by commenting on the size of the terms suppressed by powers of $x_{h t}$ with respect to the $y_{t}^{4} g_{s}^{2}$ and $y_{t}^{6}$ contribution in eqs. (2.44), (2.45). While in the QCD case, eq. (2.44), the $x_{h t}$ suppressed terms are indeed smaller than the $y_{t}^{4} g_{s}^{2}$ contribution, the same is not true in the Yukawa case, eq. (2.45), where the $x_{h t}$ terms are actually larger than the $y_{t}^{6}$ contribution.

\section{Extrapolating the SM up to the Planck scale}

A full NNLO computation of the Higgs potential requires three main ingredients: 1) the two-loop effective potential; 2) three-loop beta functions for all the relevant couplings; 3) two-loop matching conditions to determine the initial values of the couplings at the electroweak scale. As anticipated in the introduction, all these ingredient are now available for the QCD, Yukawa and Higgs quartic couplings. In this section we first discuss the structure of the two-loop potential and the numerical inputs at the electroweak scale, and then present the final numerical results for the stability condition in the $M_{h}-M_{t}$ plane.

\subsection{The two-loop effective potential}

The SM effective potential is known up to two-loops [28, 29]. Its explicit form in a readyto-use expression is given in the appendix. For large field values $(h \gg v)$, the potential is very well approximated by its RG-improved tree-level expression,

$$
V_{\text {eff }}^{\text {tree }}(h)=\frac{\lambda(\mu)}{4} h^{4},
$$

with $\mu=\mathcal{O}(h)$. For this reason, if we are interested only in the condition of absolute stability of the potential, we could simply study the RG evolution of $\lambda$ imposing the 
condition $\lambda(\Lambda) \geq 0$ for any value $\Lambda$ up to the Planck scale (as for instance done in [11]). Given that $\lambda$ reaches its minimum value before $M_{\mathrm{Pl}}$, independently of its initial condition at the electroweak scale, the minimum Higgs mass ensuring vacuum stability corresponds to the initial value of $\lambda$ such that at some scale $\Lambda_{0}$

$$
\lambda\left(\Lambda_{0}\right)=\beta_{\lambda}\left(\Lambda_{0}\right)=0, \quad \beta_{\lambda}=\frac{d}{d \ln \mu} \lambda(\mu) .
$$

This is indeed the condition analyzed in ref. [21]. In principle, a more accurate determination of the minimal $M_{h}$ ensuring vacuum stability is obtained taking into account the full structure of the Higgs potential at the two-loop level. In practice, the determination of $M_{h}$ obtained by the condition (3.2) differs by about $0.1 \mathrm{GeV}$ from the one determined by the absolute stability of the RG-improved two-loop potential.

In the following we are interested also in analyzing the shape of the Higgs potential close to the Planck scale and in the scale where the instability occurs (as a function of $M_{h}$ and $M_{t}$ ). To this purpose, the study of the RG evolution of $\lambda$ is not sufficient and the complete structure of the effective potential at the two-loop level plays a significant role. As pointed out in $[9,10]$, one can always define an effective coupling $\lambda_{\text {eff }}(h)$ such that for $h \gg v$ the two-loop effective potential assumes the form

$$
V_{\mathrm{eff}}(h)=\frac{\lambda_{\mathrm{eff}}(h)}{4} h^{4} .
$$

The explicit two-loop result for $\lambda_{\text {eff }}(h)$ can be easily obtained from the two-loop potential and is given in the appendix. We report here the simplified expression obtained when, in the two-loop term, we take into account only the contributions from the strong and the top Yukawa couplings ${ }^{2}[9,10]$ :

$$
\begin{aligned}
\lambda_{\text {eff }}(h)=e^{4 \Gamma(h)}\{\lambda(h) & +\frac{1}{(4 \pi)^{2}} \sum_{p} N_{p} \kappa_{p}^{2}\left(r_{p}-C_{p}\right) \\
& \left.\quad+\frac{1}{(4 \pi)^{4}} y_{t}^{4}\left[8 g_{s}^{2}\left(3 r_{t}^{2}-8 r_{t}+9\right)-\frac{3}{2} y_{t}^{2}\left(3 r_{t}^{2}-16 r_{t}+23+\frac{\pi^{2}}{3}\right)\right]\right\} .
\end{aligned}
$$

Here all couplings are evaluated at the scale determined by the field value $(\mu=h)$, the index $p$ runs over particle species, $N_{p}$ counts degrees of freedom (with a minus sign for fermions), the field-dependent mass squared of species $p$ is $m_{p}^{2}(h)=\mu_{p}^{2}+\kappa_{p} h^{2}$ and $C_{p}$ is a constant. The values of $\left\{N_{p}, C_{p}, \mu_{p}^{2}, \kappa_{p}\right\}$ within the SM are:

\begin{tabular}{|c|ccccc|}
\hline$p$ & $t$ & $W$ & $Z$ & $h$ & $\chi$ \\
\hline$N_{p}$ & -12 & 6 & 3 & 1 & 3 \\
$C_{p}$ & $3 / 2$ & $5 / 6$ & $5 / 6$ & $3 / 2$ & $3 / 2$ \\
$\mu_{p}^{2}$ & 0 & 0 & 0 & $-m^{2}$ & $-m^{2}$ \\
$\kappa_{p}$ & $y_{t}^{2} / 2$ & $g^{2} / 4$ & $\left(g^{2}+g^{\prime 2}\right) / 4$ & $3 \lambda$ & $\lambda$ \\
\hline
\end{tabular}

\footnotetext{
${ }^{2}$ At high scales, the electroweak gauge couplings $g^{\prime}$ and $g$ become comparable in size to $y_{t}$ and $g_{s}$ (see figure 1), but their contribution to $\lambda_{\text {eff }}(h)$ turns out to be numerically small so that eq. (3.4) is a very good approximation.
} 

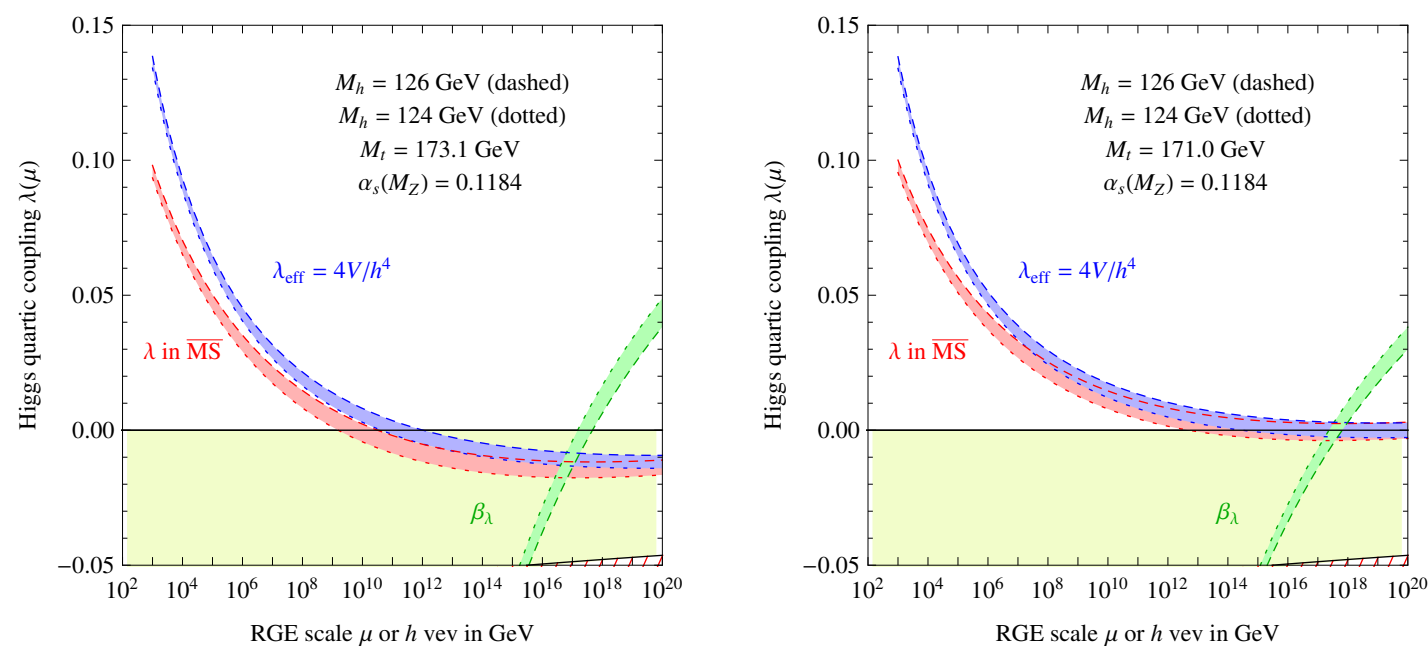

Figure 3. Evolution of the Higgs coupling $\lambda(\mu)$ and its beta function, eq. (3.2), as a function of the renormalization scale, compared to the evolution of the effective coupling $\lambda_{\text {eff }}(h)$, defined in eq. (3.3), as a function of the field value. Left: curves plotted for the best-fit value of $M_{t}$. Right: curves plotted for the lower value of $M_{t}$ that corresponds to $\lambda\left(M_{\mathrm{Pl}}\right)=0$.

The factor

$$
\Gamma(h) \equiv \int_{M_{t}}^{h} \gamma(\mu) d \ln \mu
$$

where $\gamma \equiv d \ln h / d \ln \mu$ is the Higgs field anomalous dimension, takes into account the wave-function renormalization. We have also defined $r_{p} \equiv \ln \left[\kappa_{p} e^{2 \Gamma(h)}\right]$.

The difference $\lambda_{\text {eff }}(h)-\lambda(h)$ is positive, as illustrated in figure 3 . As a result [9, 10], at a given field value the potential is more stable than what guessed from the naive expectation based on the RG-improved tree-level potential in eq. (3.1), with $\mu=h$. We finally notice that the difference $\lambda_{\text {eff }}(h)-\lambda(h)$ gets suppressed at large field values, especially when $\lambda$ reaches its minimum close to the Planck scale. This is expected according to the following two observations: 1 ) the difference between $\lambda_{\text {eff }}$ and $\lambda$ can be reabsorbed by a shift in the scales at which the two couplings are evaluated, up to finite two-loop corrections; 2) this shift has a small impact at large field values given the corresponding vanishing of $\beta_{\lambda}$ (see figure 3).

\subsection{Inputs at the electroweak scale and threshold corrections}

As far as the SM gauge couplings are concerned, we can directly use results in the literature for the couplings in the $\overline{\mathrm{MS}}$ scheme. In particular, from a global fit of electroweak precision data, performed with the additional input $M_{h} \approx 125 \mathrm{GeV}$, the following $\overline{\mathrm{MS}}$ values of the electromagnetic coupling and the weak angle renormalized at $M_{Z}$ are obtained [35]:

$$
\alpha_{\mathrm{em}}^{-1}=127.937 \pm 0.015, \quad \sin ^{2} \theta_{\mathrm{W}}=0.23126 \pm 0.00005 .
$$

From these we derive

$$
\begin{aligned}
& \alpha_{2}^{-1}\left(M_{Z}\right)=\alpha_{\mathrm{em}}^{-1} \sin ^{2} \theta_{\mathrm{W}}=29.587 \pm 0.008 \\
& \alpha_{Y}^{-1}\left(M_{Z}\right)=\alpha_{\mathrm{em}}^{-1} \cos ^{2} \theta_{\mathrm{W}}=98.35 \pm 0.013
\end{aligned}
$$


For the strong coupling we adopt

$$
\alpha_{s}\left(M_{Z}\right)=0.1184 \pm 0.0007[36]
$$

such that, including 3 loop RG running up to $M_{t}$ and matching to the theory with 6 flavors, we get

$$
g_{s}\left(M_{t}\right)=1.1645+0.0031\left(\frac{\alpha_{s}\left(M_{Z}\right)-0.1184}{0.0007}\right)-0.00046\left(\frac{M_{t}}{\mathrm{GeV}}-173.15\right) .
$$

We determine the $\overline{\mathrm{MS}}$ top-quark Yukawa coupling $\left(y_{t}\right)$ starting from the top-quark pole mass $\left(M_{t}\right)$ determined from experiments. Averaging measurements from Tevatron and LHC experiments,

$$
M_{t}= \begin{cases}173.2 \pm 0.9 \mathrm{GeV} & \text { Tevatron [37] } \\ 172.6 \pm 0.6 \pm 1.2 \mathrm{GeV} & \text { CMS } \mu j[38] \\ 174.5 \pm 0.6 \pm 2.3 \mathrm{GeV} & \text { ATLAS } \ell j[39]\end{cases}
$$

we get

$$
M_{t}=(173.1 \pm 0.7) \mathrm{GeV} .
$$

In order to translate this value into a determination of $y_{t}$ we apply: 1) QCD threshold corrections up to $O\left(\alpha_{s}^{3}\right)$ [40-42]; 2) complete one-loop electroweak corrections from ref. [43]; 3) two-loop $\mathcal{O}\left(\alpha \alpha_{s}\right)$ corrections from ref. [44], including the $\mathcal{O}\left(\alpha \alpha_{s}\right)$ terms due to the renormalization of the Fermi coupling (see section 2). As a result, we find, for the $\overline{\mathrm{MS}}$ top Yukawa coupling renormalized at the top pole mass $M_{t}$ :

$$
\begin{aligned}
y_{t}\left(M_{t}\right)= & 0.93587+0.00557\left(\frac{M_{t}}{\mathrm{GeV}}-173.15\right)-0.00003\left(\frac{M_{h}}{\mathrm{GeV}}-125\right) \\
& -0.00041\left(\frac{\alpha_{s}\left(M_{Z}\right)-0.1184}{0.0007}\right) \pm 0.00200_{\mathrm{th}} .
\end{aligned}
$$

The $\mathcal{O}\left(\alpha \alpha_{s}\right)$ term, that is the parametrically smallest correction, is equivalent to a tiny shift in $M_{t}$ below $0.1 \mathrm{GeV}$. This effect is well below the $\mathcal{O}\left(\Lambda_{\mathrm{QCD}}\right)$ irreducible non-perturbative uncertainty on the top-quark mass determined at hadron colliders (see e.g. ref. $[45,46]$ ), that is responsible for the theoretical error in eq. (3.14). More explicitly, we estimate an irreducible theoretical error of $\pm \Lambda_{\mathrm{QCD}} \approx \pm 0.3 \mathrm{GeV}$ in $M_{t}$ from non-perturbative effects, and an additional uncertainty of $\pm 0.15 \mathrm{GeV}$ from missing $\mathcal{O}\left(\alpha_{s}^{4}\right)$ threshold corrections. ${ }^{3}$

\footnotetext{
${ }^{3}$ In principle, a direct determination of the $\overline{\mathrm{MS}}$ top-quark mass at hadron colliders can be obtained from the experimental data on the $\sigma(p p / p \bar{p} \rightarrow t \bar{t})$ cross section (see ref. [47] and references therein). At present this determination leads to a value for $M_{t}$ which is perfectly consistent with eq. (3.13) but has an error four times larger [48]. For completeness and for future reference, we report here the stability condition in eq. (1.2) as a function of the MS top-quark Yukawa coupling, rather than the top-quark pole mass:
}

$$
M_{h}>129.4+2.0\left(\frac{y_{t}\left(M_{t}\right)-0.9356}{0.0054}\right)-0.35\left(\frac{\alpha_{s}\left(M_{Z}\right)-0.1184}{0.0007}\right) \pm 1.0_{\mathrm{th}}
$$



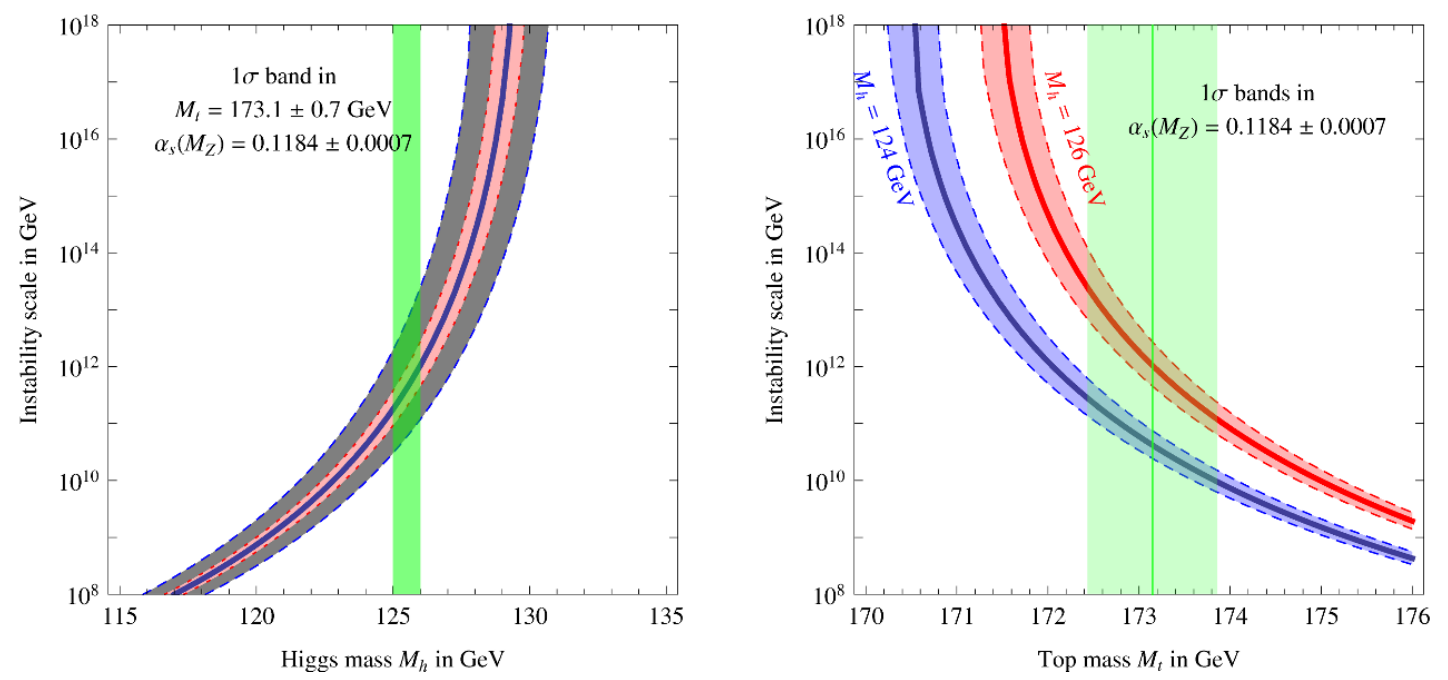

Figure 4. The instability scale $\Lambda_{I}$ at which the SM potential becomes negative as a function of the Higgs mass (left) and of the top mass (right). The theoretical error is not shown and corresponds to a $\pm 1 \mathrm{GeV}$ uncertainty in $M_{h}$.

Next, applying the threshold corrections discussed in section 2, we determine the following value for the Higgs self coupling in the $\overline{\mathrm{MS}}$ scheme renormalized at the pole top mass:

$$
\lambda\left(M_{t}\right)=0.12577+0.00205\left(\frac{M_{h}}{\mathrm{GeV}}-125\right)-0.00004\left(\frac{M_{t}}{\mathrm{GeV}}-173.15\right) \pm 0.00140_{\mathrm{th}} .
$$

The residual theoretical uncertainty, that is equivalent to an error of $\pm 0.7 \mathrm{GeV}$ in $M_{h}$, has been estimated varying the low-energy matching scale for $\lambda$ between $M_{Z}$ and $2 M_{t}$.

For completeness, we also include in the one- and two-loop RG equation the contributions of the small bottom and tau Yukawa couplings, as computed from the $\overline{\mathrm{MS}} b$-quark mass, $m_{b}\left(m_{b}\right)=4.2 \mathrm{GeV}$, and from $M_{\tau}=1.777 \mathrm{GeV}$.

\subsection{Phase diagram of the SM}

The final result for the condition of absolute stability is presented in eq. (1.2). The central value of the stability bound at NNLO on $M_{h}$ is shifted with respect to NLO computations (where the matching scale is fixed at $\mu=M_{t}$ ) by about $+0.5 \mathrm{GeV}$, whose main contributions can be decomposed as follows:

$+0.6 \mathrm{GeV}$ due to the QCD threshold corrections to $\lambda$ (in agreement with [21]);

$+0.2 \mathrm{GeV}$ due to the Yukawa threshold corrections to $\lambda$;

$-0.2 \mathrm{GeV}$ from RG equation at 3 loops (from $[19,20]$ );

$-0.1 \mathrm{GeV}$ from the effective potential at 2 loops.

As a result of these corrections, the instability scale is lowered by a factor $\sim 2$, for $M_{h} \sim$ $125 \mathrm{GeV}$, after including NNLO effects. The value of the instability scale is shown in figure 4 .

The phase diagram of the SM Higgs potential is shown in figure 5 in the $M_{t}-M_{h}$ plane, taking into account the values for $M_{h}$ favored by ATLAS and CMS data $[1,2]$. The left 

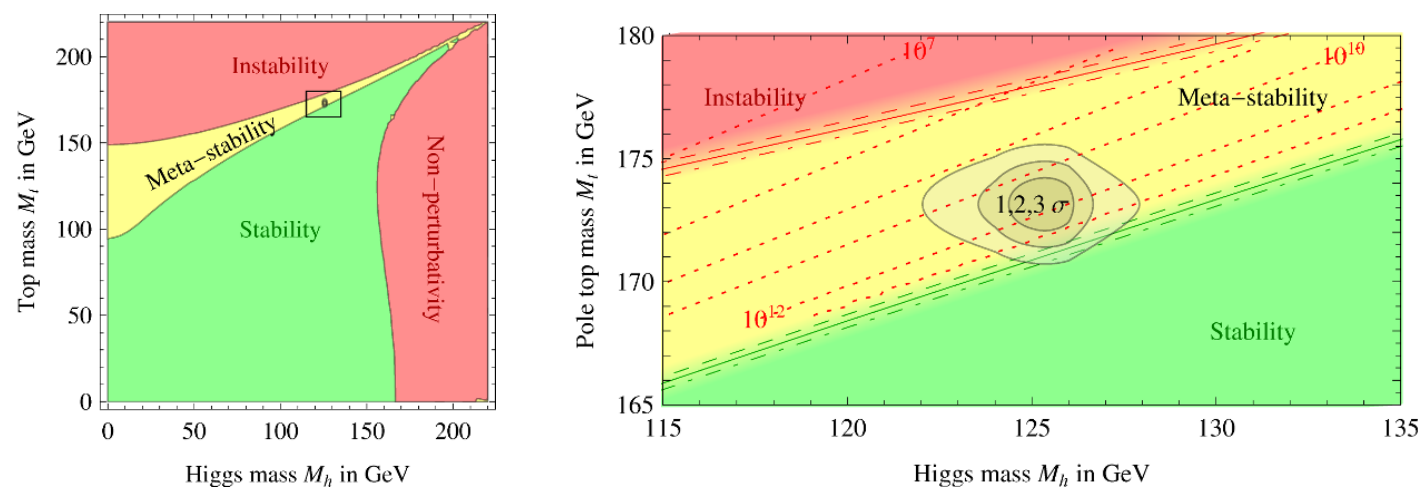

Figure 5. Regions of absolute stability, meta-stability and instability of the SM vacuum in the $M_{t}-M_{h}$ plane. Right: zoom in the region of the preferred experimental range of $M_{h}$ and $M_{t}$ (the gray areas denote the allowed region at 1,2 , and $3 \sigma$ ). The three boundaries lines correspond to $\alpha_{s}\left(M_{Z}\right)=0.1184 \pm 0.0007$, and the grading of the colors indicates the size of the theoretical error. The dotted contour-lines show the instability scale $\Lambda$ in $\mathrm{GeV}$ assuming $\alpha_{s}\left(M_{Z}\right)=0.1184$.

\begin{tabular}{|ccc|}
\hline Type of error & Estimate of the error & Impact on $M_{h}$ \\
\hline$M_{t}$ & Experimental uncertainty in $M_{t}$ & $\pm 1.4 \mathrm{GeV}$ \\
$\alpha_{\mathrm{s}}$ & Experimental uncertainty in $\alpha_{\mathrm{s}}$ & $\pm 0.5 \mathrm{GeV}$ \\
Experiment & Total combined in quadrature & $\pm 1.5 \mathrm{GeV}$ \\
\hline$\lambda$ & Scale variation in $\lambda$ & $\pm 0.7 \mathrm{GeV}$ \\
$y_{t}$ & $\mathcal{O}\left(\Lambda_{\mathrm{QCD}}\right)$ correction to $M_{t}$ & $\pm 0.6 \mathrm{GeV}$ \\
$y_{t}$ & QCD threshold at 4 loops & $\pm 0.3 \mathrm{GeV}$ \\
RGE & EW at 3 loops + QCD at 4 loops & $\pm 0.2 \mathrm{GeV}$ \\
Theory & Total combined in quadrature & $\pm 1.0 \mathrm{GeV}$ \\
\hline
\end{tabular}

Table 1. Dominant sources of experimental and theoretical errors in the computation of the SM stability bound on the Higgs mass, eq. (1.2).

plot illustrates the remarkable coincidence for which the SM appears to live right at the border between the stability and instability regions. As can be inferred from the right plot, which zooms into the relevant region, there is significant preference for meta-stability of the SM potential. By taking into account all uncertainties, we find that the stability region is disfavored by present data by $2 \sigma$. For $M_{h}<126 \mathrm{GeV}$, stability up to the Planck mass is excluded at $98 \%$ C.L. (one sided).

The dominant uncertainties in the evaluation of the minimum $M_{h}$ value ensuring absolute vacuum stability within the SM are summarized in table 1 . The dominant uncertainty is experimental and comes mostly from the measurement of $M_{t}$. Although experiments at the LHC are expected to improve the determination of $M_{t}$, the error on the top mass will remain as the largest source of uncertainty. If no new physics other than the Higgs boson is discovered at the LHC, the peculiarity of having found that the SM parameters lie at the critical border between stability and metastability regions provides a valid motivation for improved top quark mass measurements, possibly at a linear collider.

The dominant theoretical uncertainty, while reduced by about a factor of 3 with the present work, is still related to threshold corrections to the Higgs coupling $\lambda$ at the weak 

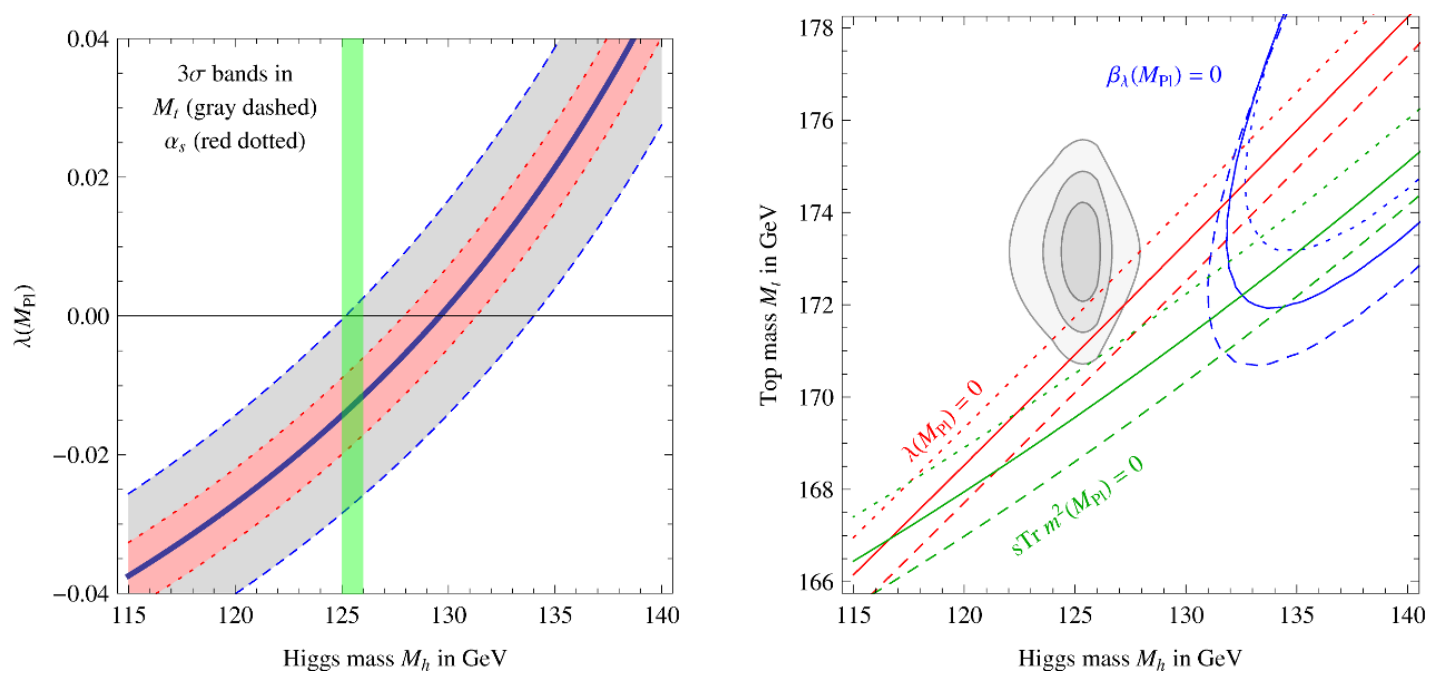

Figure 6. Left: the Higgs quartic coupling $\lambda$ at the Planck scale as a function of $M_{h}$, with $\pm 3 \sigma$ variations in $M_{t}$ and $\alpha_{\mathrm{s}}$. Right: curves in the $M_{t}-M_{h}$ plane corresponding to the conditions $\lambda\left(M_{\mathrm{Pl}}\right)=0$ (red), $\beta_{\lambda}\left(M_{\mathrm{Pl}}\right)=0$ (blue), and to the Veltman's condition of vanishing supertrace (green, see text). In all cases the dashed and dotted lines denote the $\pm 3 \sigma$ variation in $\alpha_{\mathrm{s}}$. The gray areas denote the experimentally allowed region at 1,2 , and $3 \sigma$.

scale. Another sizable theoretical uncertainty comes from the fact that the pole top mass determined at hadron colliders suffers from $\mathcal{O}\left(\Lambda_{\mathrm{QCD}}\right)$ non-perturbative uncertainties [45]. A possibility to overcome this problem and, at the same time, to improve the experimental error on $M_{t}$, would be a direct determination of the $\overline{\mathrm{MS}}$ top-quark running mass from experiments, for instance from the $t \bar{t}$ cross-section at a future $e^{+} e^{-}$collider operating above the $t \bar{t}$ threshold. In this respect, such a collider could become crucial for establishing the structure of the vacuum and the ultimate fate of our universe.

As far as the RG equations are concerned, the error of $\pm 0.2 \mathrm{GeV}$ is a conservative estimate, based on the parametric size of the missing terms. The smallness of this error, compared to the uncertainty due to threshold corrections, can be understood by the smallness of all the couplings at high scales: four-loop terms in the RG equations do not compete with finite tree-loop corrections close to the electroweak scale, where the strong and the top-quark Yukawa coupling are large.

The LHC will be able to measure the Higgs mass with an accuracy of about 100$200 \mathrm{MeV}$, which is far better than the theoretical error with which we are able to determine the condition of absolute stability.

\section{Implications}

\subsection{Boundary conditions at the Planck scale}

It is certainly a remarkable coincidence that both $\lambda$ and its beta function $\beta_{\lambda}$ nearly vanish around the Planck scale. This motivates us to explore in more detail the boundary conditions at $M_{\mathrm{Pl}}$ required to reproduce the measured values of the SM parameters. In figure 6 
(left) we show the prediction for the Higgs quartic coupling $\lambda$ at $M_{\mathrm{Pl}}$, with $3 \sigma$ bands describing the errors in $M_{t}$ and $\alpha_{s}$. For $M_{h}$ in the range preferred by LHC, $\lambda\left(M_{\mathrm{Pl}}\right)=0$ can be obtained only if $M_{t}$ is $\approx 2 \mathrm{GeV}$ below its present central value (i.e. $M_{t} \leq 171 \mathrm{GeV}$ ). However, it should be noted that $\lambda=0$ is neither a fixed point nor a point of enhanced symmetry and thus satisfying exactly this condition is not especially meaningful. The best-fit value for $\lambda$ at the Planck scale is small and negative,

$$
\lambda\left(M_{\mathrm{Pl}}\right)=-0.0144+0.0028\left(\frac{M_{h}}{\mathrm{GeV}}-125\right) \pm 0.0047_{M_{t}} \pm 0.0018_{\alpha_{s}} \pm 0.0028_{\mathrm{th}},
$$

where the uncertainties refer to the present $1 \sigma$ errors in $M_{t}$ and $\alpha_{\mathrm{s}}$, and to the theoretical error.

Equation (4.1) could be the result of matching the SM at $M_{\mathrm{Pl}}$ with a theory in which $\lambda$ vanishes at tree level and receives one-loop threshold corrections. However, the contribution to high-energy thresholds from the SM couplings at $M_{\mathrm{Pl}}$ is typically a few times $10^{-3}$. The required effect of size $10^{-2}$ can be obtained from loops of SM couplings only if these involve particles with large multiplicities, or else from loop with new (large) coupling constants. As evident from figure 1, the boundary condition of $\lambda$ at high energy is fairly independent of the precise value at which we impose it.

The right plot in figure 6 shows the dependence on $M_{h}$ and $M_{t}$ of the boundary conditions $\lambda\left(M_{\mathrm{Pl}}\right)=0$ (red line) and $\beta_{\lambda}\left(M_{\mathrm{Pl}}\right)=0$ (blue line). While $\lambda=0$ weakly depends on the scale at which is evaluated, a more pronounced dependence affects the condition $\beta_{\lambda}=0$ (see figure 3). This is because $\beta_{\lambda}$ depends not only on $\lambda$, but also on other couplings (top Yukawa and gauge) that run in the high-energy region. As a result, although $\beta_{\lambda}\left(M_{\mathrm{Pl}}\right)=0$ cannot be exactly satisfied, the beta function vanishes at scales very near the Planck mass. For instance, for $M_{t}=171.0 \mathrm{GeV}$ and $M_{h}=125 \mathrm{GeV}$, both $\lambda$ and $\beta_{\lambda}$ simultaneously vanish when they are evaluated at a scale equal to $3 \times 10^{17} \mathrm{GeV}$. In ref. [24] it was argued that $\lambda\left(M_{\mathrm{Pl}}\right) \approx 0$ and $\beta_{\lambda}\left(M_{\mathrm{Pl}}\right) \approx 0$ could be justified in the case of an asymptotically safe gravitational theory.

Just for illustration, in the right plot in figure 6 we also show the Higgs mass implied by Veltman's condition [49] that the supertrace of the squared masses of all SM particles vanishes at a given scale, here chosen to be $M_{\mathrm{Pl}}$ : $\mathrm{S} \operatorname{Tr} \mathcal{M}^{2}\left(M_{\mathrm{Pl}}\right)=0$. We remark, however, that this condition does not carry special information about the power divergences of the theory, which are dominated by UV effects, while the supertrace includes only the contribution from the IR degrees of freedom in the SM. At any rate, the possibility of a very special fine-tuning involving only the SM loop contributions and leading to $\operatorname{STr} \mathcal{M}^{2}\left(M_{\mathrm{Pl}}\right)=0$, implies a Higgs mass $M_{h} \approx(135 \pm 2.5) \mathrm{GeV}$, which is excluded at more than $3 \sigma$. Lowering the scale at which the supertrace condition is evaluated makes the disagreement even stronger.

\subsection{Higgs inflation from non-minimal coupling to gravity}

The extrapolation of the SM up to very high energy has led to some speculations about the possibility of interpreting the Higgs boson as the inflaton. One scenario for Higgs inflation [50] exploits a large non-minimal coupling between the Higgs bilinear and the Ricci scalar $R$, with an interaction Lagrangian $\xi|H|^{2} R$. The effect of this interaction is 
to flatten the Higgs potential (or any other potential) above the scale $M_{\mathrm{Pl}} / \sqrt{\xi}$, providing a platform for slow-roll inflation. A correct normalization of the spectrum of primordial fluctuations fixes the value of the coupling constant $\xi$. Using the tree-level potential, one finds $\xi \approx 5 \times 10^{4} \sqrt{\lambda}$.

This inflationary scenario, attractive for its minimality, suffers from a serious drawback. Perturbative unitarity is violated at the scale $M_{\mathrm{Pl}} / \xi$, signaling the presence of new physical phenomena associated with strong dynamics. It is naturally expected that these phenomena will affect the scalar potential above $M_{\mathrm{Pl}} / \xi$ in an uncontrollable way [51,52]. One solution is to add new degrees of freedom that restore perturbative unitarity [53, 54], although the minimality of the model is then lost. The procedure advocated by the proponents of this scenario is to assume that the strong dynamics will preserve intact the shape of the SM potential, even above $M_{\mathrm{Pl}} / \xi$. Although we find this assumption questionable, it is still interesting to address the issue of whether the Higgs data are compatible with this scenario.

A two-loop analysis of Higgs $\xi$-inflation was developed in ref. [15, 55]. The ordinary $\mathrm{SM}$ evolution is perfectly adequate below the scale $M_{\mathrm{Pl}} / \xi$, while the new interaction can affect the scalar potential at very high energy. The renormalization procedure above the inflationary scale is not unambiguous as, for instance, the renormalization scale differs in the Jordan and Einstein frames. Luckily, the slow running of $\lambda$ at high energy makes these issues irrelevant, from a practical point of view. A simple SM calculation of the potential is perfectly adequate to describe the situation of Higgs $\xi$-inflation (see also the discussion in ref. [21]).

In practice, the result is that Higgs $\xi$-inflation requires stability of the potential up to the inflationary scale $M_{\mathrm{Pl}} / \sqrt{\xi}$. As we are interested in the minimum value of the Higgs mass that satisfies this condition, the coupling $\lambda$ at the relevant scale is very small and thus the coupling $\xi$ is not particularly large, $\xi<\mathcal{O}\left(10^{3}\right)$. Therefore, the resulting restriction is stability, as given by eq. (1.2). If the LHC indication for $M_{h}=125-126 \mathrm{GeV}$ is confirmed, the simplest version of Higgs inflation is disfavored, unless the top mass is about $2 \sigma$ below its present central value. However, given the proximity of $\lambda\left(M_{\mathrm{Pl}}\right)$ to the critical value for stability, unknown one-loop threshold corrections near the Planck mass could be sufficient to rescue the proposal. It is also interesting that the introduction of a single scalar field at the scale $M_{\mathrm{Pl}} / \xi$ could simultaneously restore perturbative unitarity and cure the potential instability [56].

\subsection{Higgs inflation from false vacuum}

Alternative proposals for Higgs inflation employ the peculiarity of the SM scalar potential to develop a second minimum at large Higgs field values for a very special choice of parameters $[12,22,23]$. The possibility of using this new minimum for inflation was first contemplated in ref. [13], finding that it implies a viable prediction for the Higgs mass, but also a wrong prediction for the amplitude of density fluctuations. The latter result can be cured in non-minimal inflationary setups $[26,27]$ without affecting the prediction for the Higgs mass, which we now precisely compute. 
The first derivative of the Higgs potential $V=\lambda_{\text {eff }}(h) h^{4} / 4$ is

$$
\frac{d V}{d h}=\left(\lambda_{\mathrm{eff}}+\frac{\beta_{\mathrm{eff}}}{4}\right) h^{3} .
$$

Here $\lambda_{\text {eff }}(h)$ is the effective coupling defined in eq. (3.4) and $\beta_{\text {eff }}=d \lambda_{\text {eff }} / d \ln h$. If $\lambda_{\text {eff }}$ becomes sufficiently small, the potential can develop a minimum at $h=h_{\min }$, such that

$$
\lambda_{\text {eff }}+\left.\frac{\beta_{\text {eff }}}{4}\right|_{h=h_{\min }}=0 .
$$

This situation can occur in the proximity of a field value $h_{*}$ where $\beta_{\text {eff }}$ vanishes. In the neighborhood of $h_{*}$, we can approximate $\lambda_{\text {eff }}(h)$ as

$$
\lambda_{\text {eff }}(h) \approx \lambda_{*}+b \ln ^{2} \frac{h}{h_{*}},
$$

where $\lambda_{*}$ is the minimum value of $\lambda_{\text {eff }}$, such that $\beta_{\mathrm{eff}}\left(\lambda_{*}\right)=0$. The zero of the $\beta$ function insures that the leading log is absent in eq. (4.4) and thus $b$ is a typical two-loop coefficient. For the relevant values of the SM parameters, we find $b=0.4 /(4 \pi)^{4}$. We are interested in a situation in which the field configuration corresponds to a local minimum (while the EW vacuum remains the global minimum) and thus we want both $\lambda_{*}$ and $b$ to be positive. Using the expansion in eq. (4.4), we can compute $h_{\min }$ and the minimum of the potential $V_{\min } \equiv V\left(h_{\min }\right)$,

$$
\begin{gathered}
h_{\min } \approx h_{*} \exp \left[\frac{1}{4}\left(\sqrt{1-\frac{16 \lambda_{*}}{b}}-1\right)\right], \\
V_{\min } \approx \frac{b}{8} h_{\min }^{4} \ln \frac{h_{*}}{h_{\min }} .
\end{gathered}
$$

The minimum $h_{\min }$ exists only for extremely small values of the Higgs quartic coupling, $\lambda_{*}<b / 16$. As we vary $\lambda_{*}$ within its allowed range $\left(0<\lambda_{*}<b / 16\right)$ we find that $h_{\min }$ is always near $h_{*}\left(e^{-1 / 4}<h_{\min } / h_{*}<1\right)$, while $V_{\min }$ can change widely $\left(0<V_{\min }<b h_{\min }^{4} / 32\right)$.

If the Higgs field is trapped in the false vacuum during the early universe, it can cause inflation. The normalization of the spectrum of primordial perturbations, which is determined by $V_{\min }$, can be appropriately selected by tuning the ratio $\lambda_{*} / b$. The main difficulty of this scenario is to achieve a graceful exit from the inflationary phase. Two mechanisms have been proposed. The first one [26] employs a new scalar field, non-minimally coupled to gravity, that slows down the expansion rate, thus allowing for quantum tunneling of the Higgs out of the false vacuum. The second mechanism [27] uses a scalar field weakly coupled to the Higgs which, during the cosmological evolution, removes the barrier in the Higgs potential in a process analogous to hybrid inflation. So, in practice, the minimality of the SM is lost and one may wonder if there is any conceptual gain with respect to adding a new scalar playing the role of the inflaton. Nevertheless, it is interesting to investigate whether the Higgs and top masses are compatible with the intriguing possibility of a false vacuum at large field value.

In practice the above equations amount to saying that the conditions for the existence of a second (unstable) vacuum are that $\lambda_{\text {eff }}$ (essentially) vanishes at the same scale at 

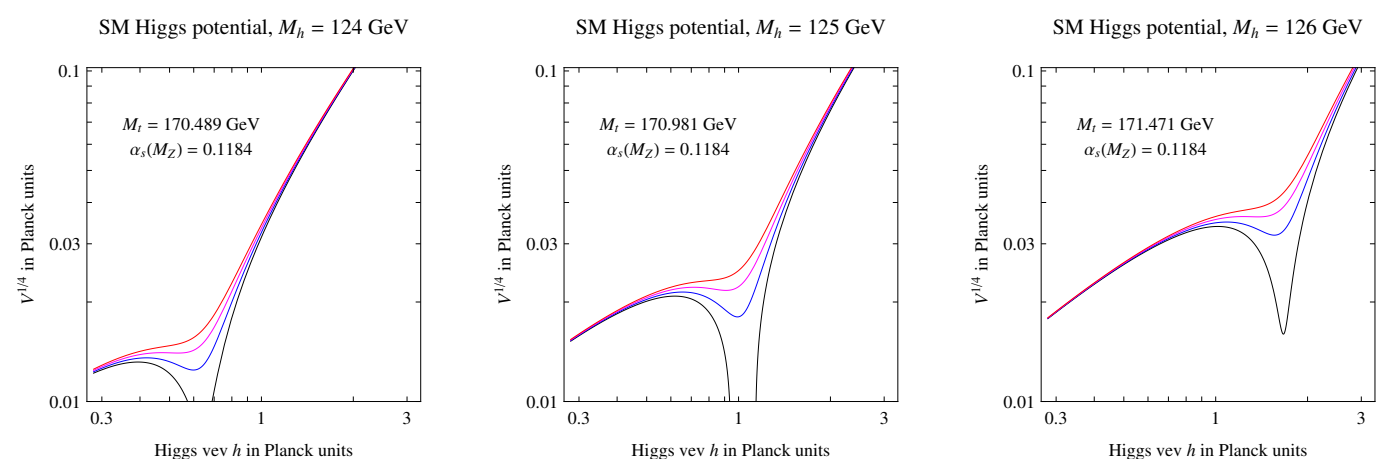

Figure 7. Two-loop SM Higgs potential for $M_{h}=124,125,126 \mathrm{GeV}$ around the critical top mass that gives a second minimum around the Planck scale. The various curves correspond to variations in $M_{t}$ by $0.1 \mathrm{MeV}$.

which $\beta_{\text {eff }}$ vanishes. This corresponds to the intersection between the red band (condition $\lambda \approx \lambda_{\text {eff }}=0$ ) and the blue band (condition $\beta_{\lambda}=0$ ) in figure 6(right). It is remarkable that the SM can achieve these conditions, although they require a top mass about $2 \sigma$ below the central value. The resulting relation between $M_{h}$ and $M_{t}$ corresponds to the equality in eq. (1.2), and is precisely studied in figure 7 where we compute for $M_{h}=$ $\{124,125,126\} \mathrm{GeV}$ the predicted top mass and show the shape of the potential around the false vacuum. The value of $V_{\min }$ can be changed by tuning $\lambda_{*}$ or, in other words, by accurate variations of $M_{h}$ and $M_{t}$. The existence of the false vacuum depends critically on the exact values of the SM parameters and requires dialing $M_{h}$ and $M_{t}$ by one part in $10^{6}$. However, the exact value of the needed top mass has a theoretical uncertainty, reduced down to $\pm 0.5 \mathrm{GeV}$ thanks to our higher-order computation. Note from figure 7 that the field value where the false vacuum is positioned is larger than what was reported in $[13,26]$. The corrections in eq. (3.4) $[9,10,12]$ are mostly responsible for the larger field values found in our analysis.

\subsection{Supersymmetry}

Our higher order computation of the relation between the Higgs mass and the Higgs quartic coupling $\lambda$ has implications for any model that can predict $\lambda$. If supersymmetry is present at some scale $\tilde{m}$, then in the minimal model one finds the tree-level relation

$$
\lambda(\tilde{m})=\frac{1}{8}\left[g^{2}(\tilde{m})+g^{\prime 2}(\tilde{m})\right] \cos ^{2} 2 \beta .
$$

A dedicated analysis of the resulting prediction for the Higgs mass as function of $\tilde{m}$ and of $\tan \beta$ was performed in [57] (see also [58]). We here update the results, including the new correction which increases the predicted Higgs mass by an amount that changes with $M_{h}$. Once more, the main impact of our calculation is the reduction of the theoretical uncertainty from $\pm 3 \mathrm{GeV}$ down to $\pm 1 \mathrm{GeV}$. As a consequence, supersymmetry broken at the Planck scale, which requires $\lambda \geq 0$ and thus the stability condition eq. (1.2), is disfavored at $2 \sigma$, unless thresholds at $M_{\mathrm{Pl}}$ (or non-minimal couplings) account for the small 
Predicted range for the Higgs mass

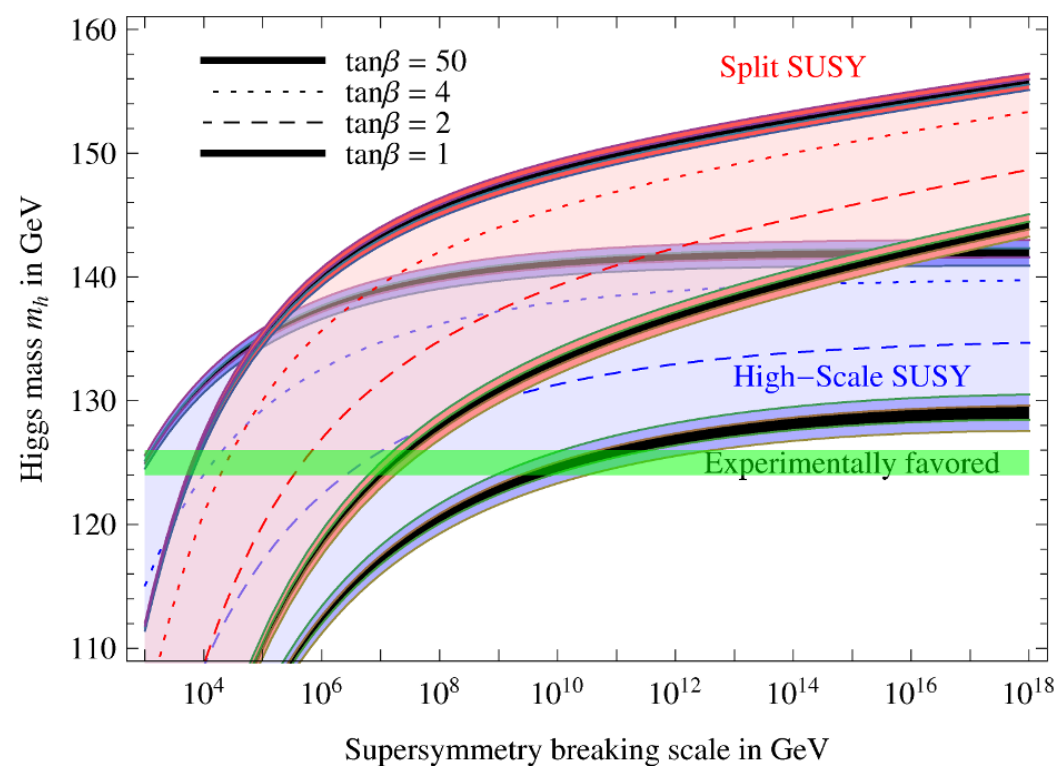

Figure 8. NNLO prediction for the Higgs mass $M_{h}$ in High-Scale Supersymmetry (blue, lower) and Split Supersymmetry (red, upper) for $\tan \beta=\{1,2,4,50\}$. The thickness of the lower boundary at $\tan \beta=1$ and of the upper boundary at $\tan \beta=50$ shows the uncertainty due to the present $1 \sigma$ error on $\alpha_{s}$ (black band) and on the top mass (larger colored band).

negative value of $\lambda\left(M_{\mathrm{Pl}}\right)$, see eq. (4.1). Thresholds at one loop have been computed in [57] including finite parts and the constant factor due to the fact that the $\overline{\mathrm{MS}}$ renormalization scheme breaks supersymmetry. A simplified, but illustrative, formula for the supersymmetric threshold corrections is obtained by taking a common mass $M_{2}$ for weak gauginos and higgsinos, and a common mass $m_{\tilde{t}}$ for the stops,

$$
\delta \lambda\left(M_{\mathrm{Pl}}\right) \approx \frac{1}{(4 \pi)^{2}}\left[-0.25+0.12 \ln \frac{m_{\tilde{t}}}{M_{2}}+0.05 \ln \frac{m_{A}}{M_{2}}\right],
$$

where $m_{A}$ is the mass of the Higgs pseudoscalar. The absence of a scale dependence in eq. (4.8) is a consequence of the approximate cancellation of $\beta_{\lambda}$ around $M_{\mathrm{Pl}}$. Negative values of the boundary condition for $\lambda$ thus require stops lighter than higgsinos, winos, and bino. By using the full formula and allowing for each sparticle mass to vary by one order of magnitude above or below the average mass $\tilde{m}$ we find

$$
-0.006<\lambda\left(M_{\mathrm{Pl}}\right)<0.002 .
$$

This is insufficient to reach the central value of eq. (4.1) and thus indicates that supersymmetry has to be broken at a scale below $M_{\mathrm{Pl}}$, if the Higgs mass has to match a supersymmetric boundary condition.

Our predictions for the Higgs mass as a function of the supersymmetry breaking scale $\tilde{m}$ are illustrated in figure 8, in the case of High-Scale Supersymmetry (all supersymmetric particles with masses equal to $\tilde{m}$ ) and Split Supersymmetry [59-61] (supersymmetric scalars 
with masses equal to $\tilde{m}$ and supersymmetric fermions at the weak scale). We refer the reader to ref. [57] for details about the underlying assumptions of the calculation.

Figure 8 shows not only how $M_{h} \approx 125 \mathrm{GeV}$ disfavors supersymmetry broken at a very high scale, but also the well know fact that the usual scenario of weak-scale supersymmetry can account for the Higgs mass only for extreme values of the parameters (such as large $\tan \beta$, heavy stops, maximal stop mixing). In the case of Split Supersymmetry, large values of $\tilde{m}$ are clearly excluded by LHC Higgs searches. On the other hand, (mildly "unnatural") scenarios in which the masses of supersymmetric scalars are one-loop larger than the weak scale [62-64] are in good agreement with data.

\section{Conclusions}

One of the most important questions addressed by the LHC is naturalness. Not only will the answer affect our understanding of the mechanism for EW breaking, but it will also determine our strategy for future directions in theoretical physics. On one side we have the avenue of larger symmetries unifying physical laws in a single fundamental principle; on the other side we have new kinds of paradigms, where parameters are not understood by naturalness arguments in the context of well-defined effective theories. At present, the multiverse is one of the most intriguing options to pursue the latter path.

If the LHC finds Higgs couplings deviating from the SM prediction and new degrees of freedom at the $\mathrm{TeV}$ scale, then the most important question will be to see if a consistent and natural (in the technical sense) explanation of EW breaking emerges from experimental data. But if the LHC discovers that the Higgs boson is not accompanied by any new physics, then it will be much harder for theorists to unveil the underlying organizing principles of nature. The multiverse, although being a stimulating physical concept, is discouragingly difficult to test from an empirical point of view. The measurement of the Higgs mass may provide a precious handle to gather some indirect information.

Once we extrapolate the SM to very short distances, we find that the values of the Higgs mass, hinted by the first LHC results $(125-126 \mathrm{GeV})$, lie right at the edge between EW stability and instability regions, see figure 5 . Moreover, the slow running of the quartic Higgs coupling $\lambda$ in the high-energy regime implies that the instability scale most critically depends on the Higgs mass $M_{h}$. A small change in $M_{h}$ (and $M_{t}$ ) can drastically modify our conclusions regarding vacuum stability. This special situation motivated us to perform a NNLO calculation of the Higgs potential in the SM, which is the subject of this paper.

Our calculation includes three-loop running for gauge, top Yukawa, and Higgs quartic coupling and two-loop matching conditions keeping the leading effects in $\alpha_{s}, y_{t}$ and $\lambda$. In particular, we have computed for the first time the two-loop threshold correction to $\lambda$, which was the most sizable missing ingredient of the NNLO result. The completion of the NNLO calculation allows us to reduce the theoretical error in the stability limit on the Higgs mass from $3 \mathrm{GeV}$ to $1 \mathrm{GeV}$. Our final result is shown in eq. (1.2). After our calculation, the largest source of uncertainty comes from the parametric dependence on the top quark mass, which leads to a $1 \sigma$ error of $1.4 \mathrm{GeV}$ in the critical Higgs mass. Of course our calculation reliably accounts for IR effects, but ignores possible new (unknown) UV threshold effects 
occurring near the Planck scale. Since our goal is to learn information about physics at very short distances, the high-energy corrections constitute an opportunity, rather than a deficiency in the calculation.

The first lesson that we learn from the SM extrapolation is that the Higgs mass hinted by LHC results corresponds to $\lambda \approx 0$ and $\beta_{\lambda} \approx 0$ at high energies. This, by itself, is an intriguing result because $\lambda=0$ is the critical value for stability and it may hide some information about Planckian physics. With our precise calculation, we can investigate further the situation. We find that, for $M_{h}=125 \mathrm{GeV}, \lambda\left(M_{\mathrm{Pl}}\right)=-0.014 \pm 0.006$, see eq. (4.1). The exact vanishing of $\lambda\left(M_{\mathrm{Pl}}\right)$ is excluded at $2 \sigma$. Moreover, the smallness of $\beta_{\lambda}$ at high energy implies that $\lambda$ remains negative in a relatively large energy range. Indeed, we find that, for $M_{h}=125 \mathrm{GeV}$, the instability scale develops at $10^{11 \pm 1} \mathrm{GeV}$. Quantum tunneling is sufficiently slow to ensure at least metastability of the EW vacuum, see figure 5 . The small value of $\lambda\left(M_{\mathrm{Pl}}\right)$ may indicate a radiative origin, although typical one-loop effects of SM couplings appears to be insufficient to account for it. We have also shown that $\beta_{\lambda}$ varies more rapidly at high energy and vanishes at a scale of about $3 \times 10^{17} \mathrm{GeV}$.

The stability of the SM potential is a crucial issue for models of inflation that employ the Higgs boson. We have analyzed several proposals showing that present data disfavor them at $98 \%$ C.L. These models can still be viable if the top quark mass turns out to be less than about $171 \mathrm{GeV}$ or if new physics around $M_{\mathrm{Pl}}$ slightly modifies the shape of the Higgs potential. The latter possibility, although fairly plausible, limits the predictability and the minimality of the approach. We have also updated previous predictions for the Higgs mass in High-Scale Supersymmetry and Split Supersymmetry.

It is natural to try to speculate on the possible meaning of the near vanishing of $\lambda$ and $\beta_{\lambda}$ around the Planck scale. The coupling $\lambda=0$ is the critical value that separates the ordinary EW phase from a phase in which the Higgs field slides to very large values. It is noteworthy that the hierarchy problem can also be interpreted as a sign of near criticality between two phases [65]. The coefficient $m^{2}$ of the Higgs bilinear in the scalar potential is the order parameter that describes the transition between the symmetric phase $\left(m^{2}>0\right)$ and the broken phase $\left(m^{2}<0\right)$. In principle, $m^{2}$ could take any value between $-M_{\mathrm{Pl}}^{2}$ and $+M_{\mathrm{Pl}}^{2}$, but quantum corrections push $m^{2}$ away from zero towards one of the two end points of the allowed range. The hierarchy problem is the observation that in our universe the value of $m^{2}$ is approximately zero or, in other words, sits near the boundary between the symmetric and broken phases. Therefore, if the LHC result is confirmed, we must conclude that both $m^{2}$ and $\lambda$, the two parameters of the Higgs potential, happen to be near critical lines that separate the EW phase from a different (and inhospitable) phase of the SM. We do not know if this peculiar quasi-criticality of the Higgs parameters is just a capricious numerical coincidence or the herald of some hidden truth.

The occurrence of criticality could be the consequence of symmetry. For instance, supersymmetry implies $m^{2}=0$. If supersymmetry is marginally broken, $m^{2}$ would remain near zero, solving the hierarchy problem. But if no new physics is discovered at the LHC, we should turn away from symmetry and look elsewhere for an explanation of the nearcriticality of $m^{2}$.

The critical value $\lambda=0$ could be justified by symmetry reasons. For instance, if the 
Higgs is a Goldstone boson, its potential vanishes and both $m^{2}$ and $\lambda$ are zero. The nonvanishing top Yukawa coupling prevents this possibility to be realized exactly. Radiative corrections then completely spoil the solution to the hierarchy problem, but could generate a small and negative value of $\lambda$ at the Planck scale, compatible with our results. Supersymmetry broken at high scales could also account for a vanishing boundary condition of $\lambda$, if $\tan \beta=1$. However, we have shown that, unless the Higgs has strong couplings with new states that live at Planckian energy, the supersymmetric boundary condition cannot be satisfied at $M_{\mathrm{Pl}}$, see figure 8 .

Alternatively, criticality could be the consequence of dynamics. If transplanckian dynamics induce a large anomalous dimension for the Higgs field, the matching condition at $M_{\mathrm{Pl}}$ of the quartic coupling $\lambda$ could be very small, while the top Yukawa coupling remains sizable. It was argued in ref. [24] that gravity itself could be responsible for a large anomalous dimension of the Higgs in the transplanckian region.

It is known that statistical systems often approach critical behaviors as a consequence of some internal dynamics or are attracted to the critical point by the phenomenon of selforganized criticality [66]. As long as no new physics is discovered, the lack of evidence for a symmetry explanation of the hierarchy problem will stimulate the search for alternative solutions. The observation that both parameters in the Higgs potential are quasi-critical may be viewed as evidence for an underlying statistical system that approaches criticality. The multiverse is the most natural candidate to play the role of the underlying statistical system for SM parameters. If this vision is correct, it will lead to a new interpretation of our status in the multiverse: our universe is not a special element of the multiverse where the parameters have the peculiarity of allowing for life, but rather our universe is one of the most common products of the multiverse because it lies near an attractor critical point. In other words, the parameter distribution in the multiverse, instead of being flat or described by simple power laws (as usually assumed) could be highly peaked around critical lines because of some internal dynamics. Rather than being selected by anthropic reasons, our universe is simply a very generic specimen in the multitude of the multiverse.

The indication for a Higgs mass in the range $125-126 \mathrm{GeV}$ is the most important result from the LHC so far. If no new physics at the $\mathrm{TeV}$ scale is discovered, it will remain as one of the few and precious handles for us to understand the governing principles of nature. The apparent near criticality of the Higgs parameters may then contain information about physics at the deepest level.

\section{Acknowledgments}

J.R.E. thanks D.R.T. Jones and M. Steinhauser for useful information exchanges. J.R.E and J.E.-M. thank CERN for hospitality and partial financial support. G.D. thanks P. Slavich for useful discussions. This work was supported by the ESF grant MTT8; by SF0690030s09 project; by the EU ITN "Unification in the LHC Era", contract PITN- GA2009-237920 (UNILHC); the Spanish Ministry MICINN under contracts FPA2010-17747 and FPA2008-01430; the Spanish Consolider-Ingenio 2010 Programme CPAN (CSD200700042); and the Generalitat de Catalunya grant 2009SGR894; by the Research Executive 
Agency (REA) of the European Union under the Grant Agreement number PITN-GA2010-264564 (LHCPhenoNet).

\section{A SM effective potential up to two-loops}

The SM effective potential is known up to two-loops [28, 29]. We present here its explicit expression in the $\overline{\mathrm{MS}}$ scheme and the Landau gauge. The tree-level part is (in this section we denote the Higgs field by $\phi$ to avoid confusion with the symbol $h$ defined later)

$$
V_{0}(\phi)=-\frac{1}{2} m^{2} \phi^{2}+\frac{1}{4} \lambda \phi^{4} .
$$

The one-loop Coleman-Weinberg potential [67] is

$$
\begin{gathered}
V_{1}(\phi)=\frac{\kappa}{4}\left[-12 m_{t}^{4}\left(L_{t}-3 / 2\right)+6 m_{W}^{4}\left(L_{W}-5 / 6\right)+3 m_{Z}^{4}\left(L_{z}-5 / 6\right)\right. \\
\left.+m_{h}^{4}\left(L_{h}-3 / 2\right)+3 m_{\chi}^{4}\left(L_{\chi}-3 / 2\right)\right]
\end{gathered}
$$

where $\kappa=1 /\left(16 \pi^{2}\right), m_{t}^{2}=y_{t}^{2} \phi^{2} / 2$ is the top mass squared, $m_{h}^{2}=-m^{2}+3 \lambda \phi^{2}$ the Higgs mass squared, $m_{\chi}^{2}(\phi)=-m^{2}+\lambda \phi^{2}$ is the Goldstone mass squared and $L_{t}=\ln \left(m_{t}^{2} / Q^{2}\right)$, etc.

We split the two-loop potential in different pieces according to their diagrammatic origin. We use the short-hand notation $t \equiv m_{t}^{2}, w \equiv m_{w}^{2}, z \equiv m_{z}^{2}, h \equiv m_{h}^{2}, \chi \equiv m_{\chi}^{2}$ and we neglect the bottom Yukawa coupling. The important top Yukawa contribution is

$$
V_{Y}=\frac{3}{2} y_{t}^{2} \kappa^{2}\left[2 J_{t t}-4 J_{t g}-2 J_{t h}+(4 t-h) I_{t t h}+2(t-\chi) I_{t g 0}-\chi I_{t t z}\right]
$$

There is a purely scalar piece

$$
V_{S}=\frac{3}{4} \kappa^{2} \lambda\left[5 J_{g g}+2 J_{h g}+J_{h h}-4 \lambda \phi^{2}\left(I_{h g g}+I_{h h h}\right)\right],
$$

a purely gauge part

$$
\begin{aligned}
V_{V}= & \frac{e^{2}}{4 z} \kappa^{2}(z-w)\left[J_{z w}+w\left(I_{z w 0}-I_{w 00}\right)\right] \\
& -\frac{e^{2}}{4} \kappa^{2} w\left[2\left(11 A_{z}-25 A_{w}\right)+\frac{1}{w}\left(24 J_{w w}+25 J_{z w}\right)+24 I_{z w w}+10 I_{z w 0}-9 I_{w 00}+49 w\right] \\
& +\frac{g^{2}}{4} \kappa^{2} w\left[\frac{58}{3}\left(A_{z}+2 A_{w}\right)+\frac{1}{w}\left(7 J_{w w}+15 J_{z w}\right)+58 I_{z w w}-9 I_{z w 0}+I_{z 00}+I_{w 00}+76 w\right] \\
& +\frac{G^{2}}{8} \kappa^{2}\left[J_{w w}-(16 w+z) I_{z w w}+2(8 w+z) I_{z w 0}-z I_{z 00}+4 w^{2}\right]
\end{aligned}
$$

a fermion-gauge boson part $^{4}$ (which includes the important QCD piece)

$$
V_{F V}=8 g_{s}^{2} \kappa^{2} m_{t}^{4}\left(3 L_{t}^{2}-8 L_{t}+9\right)+\frac{16}{3} e^{2} \kappa^{2}\left(t A_{z}+J_{t z}-t I_{t t 0}+t I_{t t z}\right)
$$

\footnotetext{
${ }^{4}$ Notice that the first paper in ref. [28, 29] contains a typo for this piece, with an extra factor 3 for the lepton-lepton- $Z$ contributions.
} 


$$
\begin{aligned}
& +\frac{g^{2}}{6} \kappa^{2}\left\{9 t^{2}-16 t w-36 w^{2}-26 t A_{t}+6(4 w-3 t) A_{w}+8(t+4 w) A_{z}-4 J_{t t}+8 J_{t z}\right. \\
& \left.+8\left[(t-2 w) I_{t t z}-6 w I_{w 00}-10 w I_{z 00}\right]+\frac{9}{w}\left[(t-2 w) J_{t w}+(t-w)(t+2 w) I_{t w 0}-t^{2} I_{t 00}\right]\right\} \\
& +\frac{G^{2}}{6} \kappa^{2}\left\{-t A_{t}-(17 t+40 w-20 z) A_{z}+\frac{17}{2} J_{t t}-17 J_{t z}-\frac{1}{2}(7 t-40 w+17 z) I_{t t z}\right. \\
& \left.+\left(100 w-\frac{103}{2} z\right) I_{z 00}+9 t^{2}+20 t w-48 w^{2}-4 t z+60 w z-30 z^{2}\right\}
\end{aligned}
$$

and a scalargauge boson part

$$
\begin{aligned}
V_{S V}=g^{2} \kappa^{2}\left\{\left[\frac{1}{2}(h+3 \chi+z)-\frac{1}{3} w\right] A_{w}+\frac{3}{2} w\left(A_{h}+A_{g}\right)+\frac{1}{4}\left(J_{g g}+J_{h g}\right)+\frac{(h-w)^{2}}{4 w} I_{w h 0}\right. \\
+\frac{1}{4 w}\left[\frac{1}{2}(h-2 w) J_{w w}+(3 w+\chi-h) J_{w h}+(h+5 w+z-\chi) J_{w g}\right]-\left(\frac{w}{4}-\chi\right) I_{w g g} \\
\left.\quad-\frac{1}{8 w}\left(h^{2}-4 h w+12 w^{2}\right) I_{w w h}-\left[\frac{1}{4 w}(h+w-\chi)^{2}-h\right] I_{w h g}-w\left(w+\frac{h}{2}\right)\right\} \\
+\frac{1}{2}\left\{\begin{array}{l}
w \leftrightarrow z \\
g \leftrightarrow G
\end{array}\right\}+\frac{3 g^{2}}{16 w} \kappa^{2}\left(8 \lambda^{2} \phi^{4} I_{h g 0}-h^{2} I_{h 00}\right) \\
-\frac{e^{2}}{4 w z}(z-w) \kappa^{2}\left\{(w+z-\chi) J_{z w}-w J_{z g}+\left[(w+z-\chi)^{2}+8 w z\right] I_{z w g}\right. \\
\left.\quad-(w-\chi)^{2} I_{w g 0}-(z-\chi)^{2} I_{z g 0}+\chi^{2} I_{g 00}\right\} \\
-\frac{e^{2}}{2} \kappa^{2}\left\{\left(4 \chi+w-\frac{5}{3} z-\frac{z^{2}}{2 w}\right) A_{z}+w A_{W}-J_{w g}+\left(4-\frac{z}{4 w}\right) J_{z g}+\frac{3}{2} \chi^{2}+2 z(z+\chi)\right. \\
\left.\quad-\frac{w}{2}(w+2 \chi)-\frac{1}{4} \chi^{2}\left(6+\pi^{2}\right)+(4 \chi-z) I_{z g g}-\frac{13}{4} \chi I_{g g 0}+\frac{3}{2}(3 w-\chi) I_{w g 0}\right\} .
\end{aligned}
$$

The functions $A, J$ and $I$ are

$$
\begin{aligned}
A_{x} \equiv A[x] \equiv & x\left(L_{x}-1\right), \\
J_{x y} \equiv J[x, y] \equiv & A[x] A[y], \\
I_{x y z} \equiv I[x, y, z] \equiv & \frac{1}{2}\left[(x-y-z) L_{y} L_{z}+(-x+y-z) L_{x} L_{z}+(-x-y+z) L_{x} L_{y}\right] \\
& +2\left(x L_{x}+y L_{y}+z L_{z}\right)-\frac{5}{2}(x+y+z)-\frac{1}{2} \xi[x, y, z],
\end{aligned}
$$

where $L_{x}=\ln \left(x / Q^{2}\right)$ and

$$
\begin{aligned}
\xi[x, y, z]=R[ & 2 \ln \left(\frac{x-y+z-R}{2 z}\right) \ln \left(\frac{-x+y+z-R}{2 z}\right)-\ln \left(\frac{x}{z}\right) \ln \left(\frac{y}{z}\right) \\
& \left.-2 \operatorname{Li}_{2}\left(\frac{x-y+z-R}{2 z}\right)-2 \operatorname{Li}_{2}\left(\frac{-x+y+z-R}{2 z}\right)+\frac{\pi^{2}}{3}\right]
\end{aligned}
$$


where $R^{2}=x^{2}+y^{2}+z^{2}-2 x y-2 x z-2 y z$ and $\operatorname{Li}_{2}(x)$ is the dilogarithm function. The above expression is valid for $R^{2}>0$, while for $R^{2}<0$ the analytical continuation should be used instead. Some particular cases of the previous functions which are useful to evaluate the potential are

$$
\begin{aligned}
I[x, y, 0]= & (x-y)\left[\operatorname{Li}_{2}\left(\frac{y}{x}\right)-\frac{\pi^{2}}{6}-\left(L_{x}-L_{y}\right) L_{x-y}+\frac{1}{2} L_{x}^{2}\right] \\
& -\frac{5}{2}(x+y)+2 x L_{x}+2 y L_{y}-x L_{x} L_{y}, \\
I[x, x, 0]= & x\left(-L_{x}^{2}+4 L_{x}-5\right), \\
I[x, 0,0]= & x\left(-\frac{1}{2} L_{x}^{2}+2 L_{x}-\frac{5}{2}-\frac{\pi^{2}}{6}\right) .
\end{aligned}
$$

The 2-loop subleading corrections to $\lambda_{\text {eff }}$ not included in eq. (3.4), obtained from the full 2-loop correction (but setting $\lambda \rightarrow 0$, as this coupling is particularly small at high field values) are explicitly given by

$$
\begin{aligned}
\delta \lambda_{\mathrm{eff}}=\kappa^{2}\{ & \frac{g^{6}}{48}\left[-30 r_{w}^{2}-18 r_{t / w} r_{(t-w)^{2} /(t w)}+532 r_{w}+144 r_{z / w}-598+12 \pi^{2}\right) \\
& +\frac{g^{4} G^{2}}{96}\left[397-32 r_{t / z}^{2}+126 r_{z / w}^{2}+66 r_{z}^{2}+27 r_{w}^{2}-232 r_{z}-138 r_{w}+160 \frac{\pi^{2}}{3}\right] \\
& +\frac{g^{4} y_{t}^{2}}{24}\left(-27 r_{w}^{2}+27 r_{t / w} r_{(t-w)^{2} /(t w)}-100 r_{t}-128 r_{z}+36 r_{w}+333+9 \pi^{2}\right) \\
& -\frac{g^{2} G^{4}}{96}\left[219 r_{z}^{2}-40 r_{t / z}^{2}+21 r_{w / z}^{2}-730 r_{z}+6 r_{w}+715+200 \frac{\pi^{2}}{3}\right] \\
& +\frac{2}{3} G^{2} y_{t}^{4}\left(3 r_{t}^{2}-8 r_{t}+9\right)-\frac{G^{6}}{192}\left(34 r_{t / z}^{2}-273 r_{z}^{2}+3 r_{w / z}^{2}+940 r_{z}-961-206 \frac{\pi^{2}}{3}\right) \\
& +\frac{G^{4} y_{t}^{2}}{48}\left[27\left(r_{t / z}^{2}-r_{z}^{2}\right)-68 r_{t}-28 r_{z}+189\right]+\frac{5}{3} g^{2} G^{2} y_{t}^{2}\left(2 r_{t}+4 r_{z}-9\right) \\
& -\frac{3 y_{t}^{6}}{2}\left(3 r_{t}^{2}+2 r_{t / w} r_{(t-w) / t}-16 r_{t}+23+\frac{\pi^{2}}{3}\right)+\frac{3}{4}\left(g^{6}-3 g^{4} y_{t}^{2}+4 y_{t}^{6}\right) \operatorname{Li}_{2}[w / t] \\
& +\frac{y_{t}^{2}}{48}\left[\left(14 G^{2}-160 g^{2}+128 \frac{g^{4}}{G^{2}}\right) y_{t}^{2}+17 G^{4}-40 g^{2} G^{2}+32 g^{4}\right] \xi_{11 z t} \\
& \left.+\frac{g^{2}}{192}\left[3 G^{4}+4\left(12 G^{2}-51 g^{2}-36 \frac{g^{4}}{G^{2}}\right) g^{2}\right] \xi 11 z w\right\}
\end{aligned}
$$

where $\xi_{11 x y}=\xi(1,1, x / y)$,

$$
r_{p} \equiv \ln \left[\kappa_{p} e^{2 \gamma(h)}\right], \quad r_{t / w} \equiv \ln \left[\kappa_{t} / \kappa_{w}\right], r_{(t-w) / t} \equiv \ln \left[\left(\kappa_{t}-\kappa_{w}\right) / \kappa_{w}\right],
$$

and so on.

Open Access. This article is distributed under the terms of the Creative Commons Attribution License which permits any use, distribution and reproduction in any medium, provided the original author(s) and source are credited. 


\section{References}

[1] ATLAS collaboration, G. Aad et al., Combined search for the standard model Higgs boson using up to $4.9 \mathrm{fb}^{-1}$ of pp collision data at $\sqrt{\mathrm{s}}=7 \mathrm{TeV}$ with the ATLAS detector at the LHC, Phys. Lett. B 710 (2012) 49 [arXiv:1202.1408] [INSPIRE].

[2] CMS collaboration, S. Chatrchyan et al., Combined results of searches for the standard model Higgs boson in pp collisions at $\sqrt{s}=7$ TeV, Phys. Lett. B 710 (2012) 26 [arXiv: 1202.1488] [INSPIRE].

[3] N. Cabibbo, L. Maiani, G. Parisi and R. Petronzio, Bounds on the fermions and Higgs boson masses in grand unified theories, Nucl. Phys. B 158 (1979) 295 [InSPIRE].

[4] P.Q. Hung, Vacuum instability and new constraints on fermion masses, Phys. Rev. Lett. 42 (1979) 873 [INSPIRE].

[5] M. Lindner, Implications of triviality for the standard model, Z. Phys. C 31 (1986) 295 [INSPIRE].

[6] M. Sher, Electroweak Higgs potentials and vacuum stability, Phys. Rept. 179 (1989) 273 [INSPIRE].

[7] B. Schrempp and M. Wimmer, Top quark and Higgs boson masses: Interplay between infrared and ultraviolet physics, Prog. Part. Nucl. Phys. 37 (1996) 1 [hep-ph/9606386] [INSPIRE].

[8] G. Altarelli and G. Isidori, Lower limit on the Higgs mass in the standard model: An Update., Phys. Lett. B 337 (1994) 141 [InSPIRE].

[9] J. Casas, J. Espinosa and M. Quirós, Improved Higgs mass stability bound in the standard model and implications for supersymmetry, Phys. Lett. B 342 (1995) 171 [hep-ph/9409458] [INSPIRE].

[10] J. Casas, J. Espinosa and M. Quirós, Standard model stability bounds for new physics within LHC reach, Phys. Lett. B 382 (1996) 374 [hep-ph/9603227] [INSPIRE].

[11] G. Isidori, G. Ridolfi and A. Strumia, On the metastability of the standard model vacuum, Nucl. Phys. B 609 (2001) 387 [hep-ph/0104016] [InSPIRE].

[12] C. Burgess, V. Di Clemente and J. Espinosa, Effective operators and vacuum instability as heralds of new physics, JHEP 01 (2002) 041 [hep-ph/0201160] [INSPIRE].

[13] G. Isidori, V.S. Rychkov, A. Strumia and N. Tetradis, Gravitational corrections to standard model vacuum decay, Phys. Rev. D 77 (2008) 025034 [arXiv:0712.0242] [INSPIRE].

[14] N. Arkani-Hamed, S. Dubovsky, L. Senatore and G. Villadoro, (No) eternal inflation and precision Higgs physics, JHEP 03 (2008) 075 [arXiv:0801.2399] [INSPIRE].

[15] F. Bezrukov and M. Shaposhnikov, Standard model Higgs boson mass from inflation: two loop analysis, JHEP 07 (2009) 089 [arXiv:0904.1537] [INSPIRE].

[16] L.J. Hall and Y. Nomura, A finely-predicted Higgs boson mass from a finely-tuned weak scale, JHEP 03 (2010) 076 [arXiv:0910.2235] [INSPIRE].

[17] J. Ellis, J. Espinosa, G. Giudice, A. Hoecker and A. Riotto, The probable fate of the standard model, Phys. Lett. B 679 (2009) 369 [arXiv:0906.0954] [InSPIRE].

[18] J. Elias-Miro et al., Higgs mass implications on the stability of the electroweak vacuum, Phys. Lett. B 709 (2012) 222 [arXiv:1112.3022] [INSPIRE]. 
[19] L.N. Mihaila, J. Salomon and M. Steinhauser, Gauge coupling $\beta$-functions in the standard model to three loops, Phys. Rev. Lett. 108 (2012) 151602 [arXiv:1201.5868] [INSPIRE].

[20] K. Chetyrkin and M. Zoller, Three-loop $\beta$-functions for top-Yukawa and the Higgs self-interaction in the standard model, JHEP 06 (2012) 033 [arXiv:1205.2892] [INSPIRE].

[21] F. Bezrukov, M.Y. Kalmykov, B.A. Kniehl and M. Shaposhnikov, Higgs boson mass and new physics, arXiv:1205.2893 [inSPIRE].

[22] D. Bennett, H.B. Nielsen and I. Picek, Understanding fine structure constants and three generations, Phys. Lett. B 208 (1988) 275 [INSPIRE].

[23] C. Froggatt and H.B. Nielsen, Standard model criticality prediction: top mass $173 \pm 5 \mathrm{GeV}$ and Higgs mass $135 \pm 9 \mathrm{GeV}$, Phys. Lett. B 368 (1996) 96 [hep-ph/9511371] [INSPIRE].

[24] M. Shaposhnikov and C. Wetterich, Asymptotic safety of gravity and the Higgs boson mass, Phys. Lett. B 683 (2010) 196 [arXiv:0912.0208] [InSPIRE].

[25] M. Holthausen, K.S. Lim and M. Lindner, Planck scale boundary conditions and the Higgs mass, JHEP 02 (2012) 037 [arXiv:1112.2415] [INSPIRE].

[26] I. Masina and A. Notari, The Higgs mass range from standard model false vacuum inflation in scalar-tensor gravity, Phys. Rev. D 85 (2012) 123506 [arXiv:1112.2659] [INSPIRE].

[27] I. Masina and A. Notari, Inflation from the Higgs field false vacuum with hybrid potential, arXiv: 1204.4155 [INSPIRE].

[28] C. Ford, I. Jack and D. Jones, The standard model effective potential at two loops, Nucl. Phys. B 387 (1992) 373 [Erratum ibid. B 504 (1997) 551-552] [hep-ph/0111190] [INSPIRE].

[29] S.P. Martin, Two loop effective potential for a general renormalizable theory and softly broken supersymmetry, Phys. Rev. D 65 (2002) 116003 [hep-ph/0111209] [INSPIRE].

[30] S.P. Martin, Two loop scalar self energies in a general renormalizable theory at leading order in gauge couplings, Phys. Rev. D 70 (2004) 016005 [hep-ph/0312092] [INSPIRE].

[31] S.P. Martin, Evaluation of two loop selfenergy basis integrals using differential equations, Phys. Rev. D 68 (2003) 075002 [hep-ph/0307101] [INSPIRE].

[32] A. Sirlin and R. Zucchini, Dependence of the quartic coupling $H(m)$ on $M(h)$ and the possible onset of new physics in the Higgs sector of the standard model, Nucl. Phys. B 266 (1986) 389 [INSPIRE].

[33] G. Degrassi and P. Slavich, NLO QCD bottom corrections to Higgs boson production in the MSSM, JHEP 11 (2010) 044 [arXiv: 1007.3465] [inSPIRE].

[34] J. Fleischer, M.Y. Kalmykov and A. Kotikov, Two loop selfenergy master integrals on-shell, Phys. Lett. B 462 (1999) 169 [hep-ph/9905249] [INSPIRE].

[35] Particle Data Group collaboration, K. Nakamura et al., Review of particle physics, J. Phys. G 37 (2010) 075021 [INSPIRE].

[36] S. Bethke, The 2009 world average of $\alpha_{s}$, Eur. Phys. J. C 64 (2009) 689 [arXiv:0908.1135] [INSPIRE].

[37] Tevatron Electroweak Working Group, CDF and D0 collaboration, Combination of CDF and D0 results on the mass of the top quark using up to $5.8 \mathrm{fb}^{-1}$ of data, arXiv:1107.5255 [INSPIRE]. 
[38] CMS collaboration, Measurement of the top quark mass in the muon+jets channel, PAS-TOP-11-015 (2011).

[39] ALTAS collaboration, Top quark mass measurements at the ATLAS experiment, PHYS-SLIDE-2012-106 (2012).

[40] D.J. Broadhurst, N. Gray and K. Schilcher, Gauge invariant on-shell $Z_{2}$ in $Q E D, Q C D$ and the effective field theory of a static quark, Z. Phys. C 52 (1991) 111 [INSPIRE].

[41] K. Melnikov and T.v. Ritbergen, The three loop relation between the MS-bar and the pole quark masses, Phys. Lett. B 482 (2000) 99 [hep-ph/9912391] [INSPIRE].

[42] K. Chetyrkin and M. Steinhauser, The relation between the MS-bar and the on-shell quark mass at order $\alpha_{s}^{3}$, Nucl. Phys. B 573 (2000) 617 [hep-ph/9911434] [INSPIRE].

[43] R. Hempfling and B.A. Kniehl, On the relation between the fermion pole mass and MS Yukawa coupling in the standard model, Phys. Rev. D 51 (1995) 1386 [hep-ph/9408313] [INSPIRE].

[44] F. Jegerlehner and M.Y. Kalmykov, $O\left(\alpha \alpha_{s}\right)$ correction to the pole mass of the $t$ quark within the standard model, Nucl. Phys. B 676 (2004) 365 [hep-ph/0308216] [INSPIRE].

[45] A.H. Hoang and I.W. Stewart, Top mass measurements from jets and the Tevatron top-quark mass, Nucl. Phys. Proc. Suppl. 185 (2008) 220 [arXiv:0808.0222] [INSPIRE].

[46] S. Fleming, A. H. Hoang, S. Mantry and I.W. Stewart, Vacuum instability and new constraints on fermion masses, Phys. Rev. D 77 (2008) 074010 [hep-ph/0703207] [INSPIRE]

[47] S. Moch, P. Uwer and A. Vogt, On top-pair hadro-production at next-to-next-to-leading order, Phys. Lett. B 714 (2012) 48 [arXiv:1203.6282] [INSPIRE].

[48] S. Alekhin, A. Djouadi and S. Moch, The top quark and Higgs boson masses and the stability of the electroweak vacuum, arXiv: 1207.0980 [INSPIRE].

[49] M. Veltman, The infrared-ultraviolet connection, Acta Phys. Polon. B 12 (1981) 437 [INSPIRE].

[50] F. Bezrukov and M. Shaposhnikov, The standard model Higgs boson as the inflaton, Phys. Lett. B 659 (2008) 703 [arXiv:0710.3755] [INSPIRE].

[51] C. Burgess, H.M. Lee and M. Trott, Power-counting and the validity of the classical approximation during inflation, JHEP 09 (2009) 103 [arXiv:0902.4465] [INSPIRE].

[52] J. Barbon and J. Espinosa, On the naturalness of Higgs inflation, Phys. Rev. D 79 (2009) 081302 [arXiv:0903.0355] [INSPIRE].

[53] R.N. Lerner and J. McDonald, A unitarity-conserving Higgs inflation model, Phys. Rev. D 82 (2010) 103525 [arXiv: 1005.2978] [INSPIRE].

[54] G.F. Giudice and H.M. Lee, Unitarizing Higgs inflation, Phys. Lett. B 694 (2011) 294 [arXiv: 1010.1417] [INSPIRE].

[55] A. De Simone, M.P. Hertzberg and F. Wilczek, Running inflation in the standard model, Phys. Lett. B 678 (2009) 1 [arXiv:0812.4946] [INSPIRE].

[56] J. Elias-Miro, J.R. Espinosa, G.F. Giudice, H.M. Lee and A. Strumia, Stabilization of the electroweak vacuum by a scalar threshold effect, JHEP 06 (2012) 031 [arXiv: 1203.0237] [INSPIRE]. 
[57] G.F. Giudice and A. Strumia, Probing high-scale and split supersymmetry with Higgs mass measurements, Nucl. Phys. B 858 (2012) 63 [arXiv:1108.6077] [INSPIRE].

[58] M. Cabrera, J. Casas and A. Delgado, Upper bounds on superpartner masses from upper bounds on the Higgs boson mass, Phys. Rev. Lett. 108 (2012) 021802 [arXiv:1108.3867] [INSPIRE].

[59] N. Arkani-Hamed and S. Dimopoulos, Supersymmetric unification without low energy supersymmetry and signatures for fine-tuning at the LHC, JHEP 06 (2005) 073 [hep-th/0405159] [INSPIRE].

[60] G. Giudice and A. Romanino, Split supersymmetry, Nucl. Phys. B 699 (2004) 65 [Erratum ibid. B 706 (2005) 65-89] [hep-ph/0406088] [INSPIRE].

[61] N. Arkani-Hamed, S. Dimopoulos, G. Giudice and A. Romanino, Aspects of split supersymmetry, Nucl. Phys. B 709 (2005) 3 [hep-ph/0409232] [INSPIRE].

[62] G.F. Giudice, M.A. Luty, H. Murayama and R. Rattazzi, Gaugino mass without singlets, JHEP 12 (1998) 027 [hep-ph/9810442] [INSPIRE].

[63] J.D. Wells, PeV-scale supersymmetry, Phys. Rev. D 71 (2005) 015013 [hep-ph/0411041] [INSPIRE].

[64] N. Arkani-Hamed, A. Delgado and G. Giudice, The well-tempered neutralino, Nucl. Phys. B 741 (2006) 108 [hep-ph/0601041] [INSPIRE].

[65] G. Giudice and R. Rattazzi, Living dangerously with low-energy supersymmetry, Nucl. Phys. B 757 (2006) 19 [hep-ph/0606105] [INSPIRE].

[66] Self-organized criticality: an explanation of $1 / f$ noise, Phys. Rev. Lett. 59 (1987) 381 [INSPIRE].

[67] S.R. Coleman and E.J. Weinberg, Radiative corrections as the origin of spontaneous symmetry breaking, Phys. Rev. D 7 (1973) 1888 [INSPIRE]. 